\title{
Review of Designs for Haptic Data Visualization
}

\author{
Sabrina Panëels, and Jonathan C. Roberts, Member, IEEE,
}

\begin{abstract}
There are many different uses for haptics, such as training medical practitioners, teleoperation or navigation of virtual environments. This review focuses on haptic methods that display data. The hypothesis is that, haptic devices can be used to present information, and consequently the user gains quantitative, qualitative or holistic knowledge about the presented data. Not only is this useful for users who are blind or partially sighted (who can feel line graphs, for instance), but the haptic modality can be used alongside other modalities, to increase the amount of variables being presented, or to duplicate some variables to reinforce the presentation. Over the last twenty years a significant amount of research has been done in haptic data presentation; e.g. researchers have developed force-feedback line-graphs, bar-charts and other forms of haptic representations. However, previous research is published in different conferences and journals, with different application emphases. This article gathers and collates these various designs to provide a comprehensive review of designs for haptic data visualization. The designs are classified by their representation: Charts, Maps, Signs, Networks, Diagrams, Images and Tables. This review provides a comprehensive reference for researchers and learners, and highlights areas for further research.
\end{abstract}

Index Terms-Haptic data visualization, Haptics, non-visual visualization, Haptic visualization, Haptic design.

\section{INTRODUCTION}

$\mathbf{O}$ VER the last two decades there has been a mounting interest in non-visual forms of presentation. Researchers have utilized touch and tactile devices, force-feedback joysticks, sound (sonification) and even smell (olfaction) to represent information [1]. Not only can these methods be used to represent information to blind or partially sighted users, but they are useful in situations where the visual domain is either overloaded or impractical [2].

The aim of any haptic system is that a user feels and interacts with a virtual model. Hence, the haptic system may be usefully considered by three parts [3]. First a mathematical model is created that realizes the information that the user is trying to perceive. Second, haptic rendering is used to compute the forces required to realize the model. Finally, an electromechanical device that is capable of exerting forces to the user is required. If the haptic display is to represent an office room, then the model would enable the user to feel that a desk is solid and that a rubber-band is elastic.

With Haptic Data Visualization (HDV) the underlying model is more abstract and encodes, not a physical environment but, numerical values or an abstract mathematical concept. The specific aim of HDV is that the user understands

Sabrina Panëels is a $\mathrm{PhD}$ student at the School of Computing, University of Kent, Canterbury, Kent, UK. E-mail: sap28@kent.ac.uk

Jonathan C. Roberts is a Senior Lecturer at the School of Computer Science, Bangor University, Bangor, Gwynedd, Wales, UK. E-mail: j.c.roberts@bangor.ac.uk data that is being represented by the haptic model; not only does the user feel the model, but in doing so realizes value and can draw conclusions from that data. For instance, a model representing a line graph of stock market data would aid the user in understanding how the stock values change over time, whether increasing or decreasing. A user may also be able to perceive maximum or minimum values or points of crossover or inflection on the graph. Values may be realized through different ways, e.g. larger values could be mapped to high frequencies, with low values to low frequency vibrations. The design of the mapping is not necessarily obvious, consequently developers need to ideate new designs that enable effective HDV.

This article provides a comprehensive review and classification of designs for HDV; it provides a snapshot of the state of the art and thus demonstrates areas of future work. Previous review papers have focused on specific aspects of haptics such as input devices [4]-[6]; haptic rendering techniques [7]-[9]; rendering in the medical domain [10], and have focused on the application of telepresence and Virtual Reality [5], [11].

The work is categorized by the representation of the data: charts; maps; signs; networks; diagrams; tables and images. This categorization was chosen because it distinguishes the various types of design, enabling the methods to be uniquely classified. E.g., the structure of a network presentation, that details associated data nodes, is very different to geographic information that is displayed on a map in two dimensions. Furthermore, the appendix orders the references by these categories. For each different form we present the design, the technologies used and any issues or challenges with the presentation.

\section{BACKGROUND}

It is useful to consider the HDV process in three parts: model, rendering and device. Developers must carefully consider each part to present the data effectively to the user. Effective designs for HDV thus consider the whole process together. The principal differences with HDV and other uses of haptics is specifically the makeup of the model.

\section{A. The HDV model}

Creating an effective model is a principal component to HDV. The developer needs to decide what data is going to be presented; how this information will be mapped and transformed into haptic properties so as to determine how the user will perceive value; and how the user is to navigate. This process is similar to that of the dataflow paradigm of traditional visualization [12], see Figure 1. But HDV presents particular challenges to developers. First, the data needs to be processed, simplified and often idealized. For instance, it may 


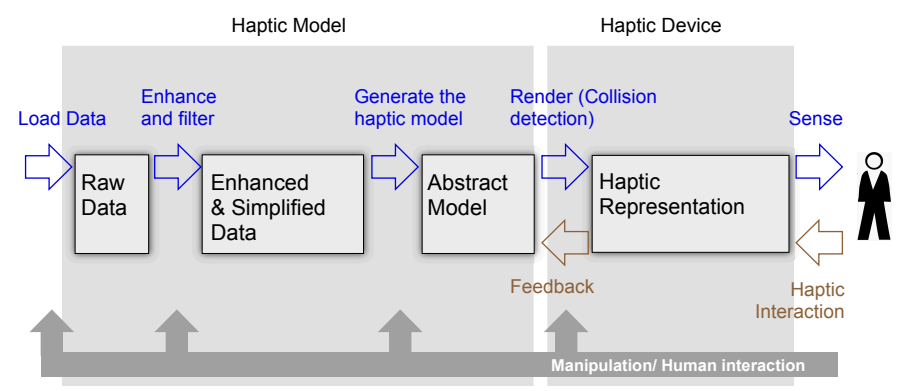

Fig. 1: Haptic Visualization Process

be that the user is interested in overall trends, in which case averages over some of the data would be more useful than the original collected values. Second, the information is then mapped to various haptic variables to build the haptic model of this information. But, there are many haptic variables that can be used to perceive information including actuator position, force-strength, vibration frequency, and surface texture. It is not often clear to a developer which mappings should be used, and what the limitations of each variable are (the limitations of each variable is also specific to the device being used). Developers also need to decide how the user is going to interact with the information; many haptic interfaces rely on the user being active in the environment to perceive the information. Furthermore, users can potentially modify any parameters to effect change in the system such as to alter the filtering, mapping or viewpoint of what is being represented. This enables the haptic visualization to be dynamic, which is not possible with many technologies that enable static haptic displays (often used to feel graphs etc.) such as swell paper or bread-board configurations using pins and bands. Subsequently, the haptic model is rendered in terms of forces and/or vibrations the user can feel.

Haptic rendering is utilized to generate the desired presentation. We refer the reader to the book chapter by Basdogan and Srinivasan [13] and the book by Lin and Otaduy [14] for a more in depth look at haptic rendering. However, it broadly consists of two processes: collision detection and collision response [13]. In the particular case of a force-feedback device such as the PHANTOM ${ }^{T M}$, being an input and output device, if the user touches a solid wall from the haptic model the collision-response mechanism transmits the right force to the device to mimic a solid surface. Thus, when the user tries to push through the solid wall the device transmits a greater force. A useful survey on collision detection is by Lin and Gottschalk [15], and although published ten years ago, it provides a good overview of the main collision detection algorithms.

Haptic technologies have been around for the last 50 years; however, it was not until the 1990's that the technologies became more widely used by application engineers. The research of tactile feedback methods has been driven by various motivations: the motivation to increase accessibility for visually impaired people (i.e., to investigate better and more intuitive ways to interact with information), to enhance current interfaces (especially on mobile and small devices) and to study the tactile sense. Achieving realistic tactile feedback is a difficult task even nowadays. Various technologies and devices have been used to provide tactile feedback such as gloves with piezoceramic benders [16], solenoid with plungers [17] and pin arrays. Benali-Khoudja et al. [4] comprehensively listed and detailed them and we encourage the reader to refer to their review. Much HDV research utilizes the PHANTOM haptic interface, but many other devices have been developed and commercialized. We refer the reader to the 'Haptics Photo Gallery' [18] which has pictures and details of many haptic tools, and to the review paper by Hayward et al. [19]. The devices range from gloves with tactile or force feedback [20][22], exoskeletons providing feedback to the hand [23] or the whole arm, space balls and arms, joysticks, mice, wheels, to interfaces with wires, pens and pin arrays.

\section{B. Classification}

One could foresee various taxonomies for HDV: those that classify the forms based on the input data, the form of the output that is perceived, the sense they utilize, the task they perform or the application domain. Indeed, most of the previous review papers have classified the research by 'areas of application' [4]-[6]. Bloomfield et al. [24] on the other hand classified the techniques by tasks, in fact they categorized the research by action categories. Recent effort has been made to provide visualization taxonomies that can apply to any sense and therefore apply to multimodal applications [25], [26]. Nesbitt [25] developed a framework to support both haptic and auditory displays, but his work focuses on development rather than classification. Whereas Coomans and Timmermans' [26] taxonomy was for virtual reality interfaces. Neither of these taxonomies [25], [26] address the real importance of data visualization, which is to enable the user to realize and explore the meaning of the information.

We categorize the work by the structure of the representation. This taxonomy enables the methods to be uniquely classified into Charts, Maps, Signs, Networks, Diagrams, Images and Tables. Each of the seven categories will be further explained in the following sections.

This categorization is inspired and extended from two researchers. Bertin [27] classified visual representations into four categories, namely diagrams, networks, maps and symbols. In his classification the diagrams category included graphs and charts. Lohse et al. [28] found similar categories (graph and tables, diagrams, icons, maps and network charts) in a study involving the sorting of visual items by subjects from various backgrounds; the results were represented on a 2D graph with one dimension realizing the amount of effort needed to understand the graphic item and the other indicating whether the representation is discrete or continuous.

\section{CHARTS}

Charts are an easy way to visually represent numeric information. In the visual domain, users can easily scan the chart to spot trends, locate maximum and minimum values or drill down to locate specific values. Furthermore, specific values are retrieved through the reference to a legend. However, 


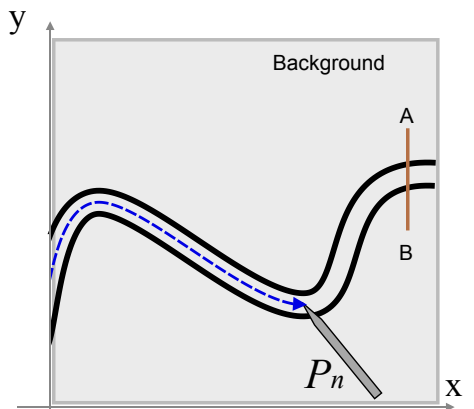

Front view

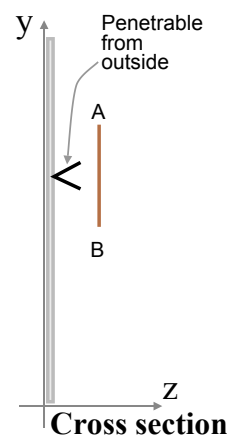

Fig. 2: Line modelling technique: engraved line as a V-shape groove attached to the background, after [31].

perceiving charts haptically is a more difficult task: users find it hard to quickly scan over the whole haptic display, and difficult to compare points to a legend to understand their value. This is due to the bandwidth of perception. Many haptic devices present the information through a point based interface; the user needs to scan the haptic device over the model to comprehend the whole display. Therefore, the challenge is to find effective representations of the numeric data in order to convey efficiently the underlying knowledge. To achieve this aim, different representations and mappings have been developed.

\section{A. Line Graphs}

Line graphs are a common representation form for presenting continuous data and are used in several domains such as mathematics, statistics, finance, etc.

1) Representation: Initially, researchers represented the line by a cylinder or an embossed ridge [29], [30]. These lines were placed on a solid background in a similar configuration to the visual graph. However, users found it difficult to keep the haptic probe on the line and they would slip out of the surface at the edges. Consequently, Fritz and Barner [29] applied attraction forces to help the user stay on the line. In 2000, in a discussion on this subject, Yu et al. [30] highlighted that embossed modelling techniques were ineffective for graph exploration and that instead engraved techniques should be used. One year later Yu et al. [31] presented an evaluation showing that users following graphs made from valleys performed better than those utilizing ridges (see Figure 2$)^{1}$.

As well as perceiving the shape of the lines, users wish to compare multiple lines, read off specific values and find extreme values. Perception of the graph line is only one part of the visualization. Much of the power of line graphs lies in displaying multiple lines on the same graph. Indeed, this enables users to compare values, analyse extremes and calculate intersections. Completing these tasks however requires the user to be able to differentiate the separate lines. One method to distinguish these lines is to use surface friction

\footnotetext{
${ }^{1}$ Readers may also be interested in the work of User Interfaces. In the real world, knobs and buttons have tactile ridges and bumps to guide the user for their use, and provide correct affordances; for example, Miller and Zeleznik [32] discuss various 3D haptic widgets, utilizing notches, dimples and infinitesimal ridges.
}

as the discriminating factor by assigning different frictions to different lines; which, was found to be effective [30], [31]. However, with multiple lines there can be intersections. Yu et al. [30] presented that although friction could help to distinguish the lines it misled the users at intersection points.

When the lines become three-dimensional they can be interpreted as 'surfaces'. Many researchers have utilized 3D haptic surfaces, but many of the papers detailing 'haptic surfaces' are about 'haptic rendering' issues, and thus out of the scope of this paper.

2) Quantitative information: Similar to visual graphs, a gridline [29], [30] was used to guide users and provide approximate coordinate values. Although Fritz and Barner [29] stated that gridlines, represented as virtual walls with just noticeable force effect, were adequate and necessary, Yu et al. [30] underlined they were rather ineffective and confusing, saying that they relied on the users' short term memory. This is because the user needs to count the gridlines and remember how many they have passed. They became confused as to which were the grid-lines and which were the graph-lines [30]. Hence, developers have sought other ways to convey values and help navigation. E.g., Fritz and Barner [29], and also Yu et al. [30] used a speech synthesizer to aid navigation.

Other haptic and sound research was carried out by Ramloll et al. [33]. Their work utilized two computers: one to render the haptics using a PHANTOM and the other to produce spatial sounds on headphones. The haptic design (like $\mathrm{Yu}$ et al. [30]) used grooves to realize the lines on the graph and friction to distinguish the lines. Values were conveyed by speech or by sound at various pitches in the following ways: (1) The Y coordinates of the line graph were mapped to pitch; (2) each curve was accompanied with a different sound, with its intensity decreasing proportionally to the distance from the listener's headphone, with the user's head at the origin (see Figure 3), (3) Speech gave the exact coordinates and an auditory representation provided an overview of the curve by playing pitches successively. This work provides the first steps towards the development of a multimodal system; but included little evaluation.

Yu et al. [34] described a similar tool that was evaluated. Their work presents an automatic online graph construction tool that can be opened in any Web browser; it used a pitch-value mapping and the users could feel the line but with the Logitech WingMan Force Feedback mouse ${ }^{T M}$. The study revealed that the multimodal representation combining audio and haptics is more effective than either modality alone. However, the auditory modality can be limiting; because users do not always have a good ear for music and feel isolated when they are using headphones.

3) Overview and Exploration: The role of an overview is to portray the general trend of the lines as well as depicting relative positions of different key elements in the chart; such as axes, the origin, inflections and any intersections. Ramloll et al. [33] and Yu et al. [34] used the auditory modality to provide this overview, and Ramloll et al. [33] guided the user to feel the most important items of the graph such as the display area, axis and curves. One challenge is to keep the user in the display area. Fritz and Barner's 3D surface 


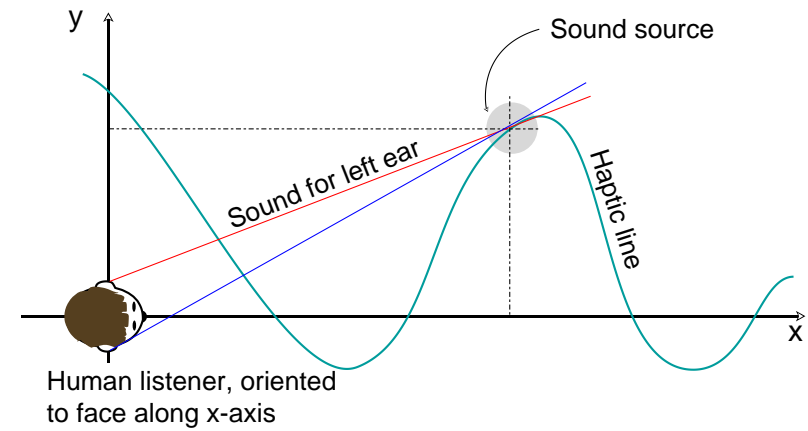

Fig. 3: Auditory design, after [33], where the user is placed virtually at the origin, facing along the $\mathrm{x}$-axis. When stereo sound is used the user is able to hear the difference between positive and negative y-values.

plots method [8] provides one solution. Their surface plot is surrounded with virtual walls (that can be removed) to prevent the user from falling off the surface. Grid planes are integrated as thin penetrable walls producing a small perceivable force. Speech is also used to dictate the user's coordinate position, while friction and texture provide haptic cues of the surface.

Roberts et al. [35], [36] also investigated the haptic overview and proposed different methodologies of exploration: unguided exploration, the constrained exploration view and the guided tour. (1) The unguided exploration enables the user to investigate anywhere in the canvas. Two solutions were tried. In the first one, the user freely explores a bumpmap model of the graph with different depths for each line in the case of multiple curves. The second solution is a 'Friction \& Zoom view' where the areas above and below the lines can be felt with different textures. (2) The constrained exploration view is a navigation interaction where a force constrains the user to follow the line. Two solutions are proposed again. The 'Independent graphs' method constrains the user to follow one line at a time where intersection points are indicated by a tactile force. The other lines can be explored by swapping the line under investigation with another one. The 'Exploded Data View' enables the user to view different and simplified views of the information such as a vertical exploration for the maxima and minima, a turning points exploration, the gradient exploration and the intersection points. (3) As for the guided tour, the user movement is constrained. Three different tour metaphors were proposed: a bus tour where the user is taken on a pre-defined route from start to end, a museum tour where the user stops at predefined points and then can investigate locally, and a water skier tour where the user has some freedom to investigate either-side of the predefined tour. Panëels and Roberts [37] and Panëels et al. [38] implemented these tour metaphors and conducted a pilot study for the single line case [37]. The number of subjects was too small to consider the results significant, however it is worth noting that users were in favour of the hypothesis that free exploration combined with the guidance-models improves the understanding of the important graph features, especially in the case of complex line graphs. Therefore more research should be conducted on providing a haptic overview.
4) Automatic translation: Many researchers have studied the automatic translation of graphics to static tactile representations, see for example, the seminal work by Way and Barner [39], [40], while not much research has been done for dynamic haptic visualization. However, Yu et al. [41] described a system that included the full process of haptic graph visualization, from the generation of the graph, its display and finally its dynamic interaction. It was developed in order to increase the accessibility to printed graphs.

The system comprised of a flatbed scanner, a PC and a force feedback device, either a PHANTOM or a WingMan mouse. First the printed graph was scanned and stored as a digital image in the computer. Then, image processing techniques were used to eliminate any irrelevant information and to extract key features for the haptic rendering. The graph was rendered using appropriate techniques, dependent on the type of force-feedback device used. The presented system only worked with simple line-graphs without intersections. Furthermore, Yu et al. [34] dealt with the automatic generation of line-graphs, bar-charts and pie-charts from random data or user-inputted data.

5) Summary: All previous studies acknowledged that haptic graphs are possible and useful but have their limitations. Many of the challenges arise from the use of single point-of-contact devices, such as the PHANTOM. Some studies [30], [33], [34] suggest a multimodal approach, using (say) audio or speech, to overcome the limitations of the device. Moreover, it is acknowledged that the haptic mapping used in these experiments is quite simple [30] or even lacking [33], [34]. Although friction was used to distinguish multiple lines [30], haptics has been limited to represent navigation. Thus, the developers have focused on the end design rather than getting across the underlying principles of the data. After all, the reason for doing a visualization is not to perceive the graphic (graph or plot) but to understand the data itself [2].

\section{B. Bar and Pie Charts}

Bar charts represent a series of categories in a set, each with values. Often the order of the categories is arbitrary, whereas the heights of the bars represent the data-values or the relative values. Visually they are efficient because a user can scan their eyes across the top of the bar chart and pre-attentively view which is the highest and lowest value being presented. Pie charts are included in this classification because they represent the same type of data entity as bar charts [42].

1) Representation: Drawing inspiration from the haptic line-graph research [30], [33], the common haptic design adopted for a bar chart is that the bars are displayed as enclosures (such to use the Logitech WingMan mouse [43]) or as engraved bars [44]. Enclosures are also used for piecharts [34]. Recently, Wall and Brewster [45] presented 'tactiles', a low-tech method to realize pie-charts. The rotary position is given by the position on the graphics-tablet, with the dynamic tactile feedback being realized by the pin-array on a VTPlayer ${ }^{2}$ tactile mouse.

\footnotetext{
${ }^{2}$ VirTouch's VTPlayer ${ }^{T M}$ is a mouse with two tactile 4-by-4 pinboard displays. www.virtouch2.com.
} 
2) Quantitative information: As opposed to line graphs, different bars or sections of a pie chart are easy to distinguish. Therefore, the main issues are: how to represent the values, how to enable comparison tasks, and how to provide an overview of the information. Yu and Brewster [43], [44] used audio alongside haptics to help the user achieve these tasks. Synthesized speech was used to provide the user with exact coordinate values, non-speech audio demonstrated the overview by mapping MIDI notes' pitch to the values, while haptics was utilized for navigation and used to trigger audio events. This is a similar design to earlier work by the same group [33]. Using this multimodal system design, Yu and Brewster conducted two comparison evaluations to prove that the multimodal approach provided better results whatever the haptic device being used. In both evaluations, the test subjects were asked to explore some charts, compare them, answer a workload questionnaire and then answer questions on the trend of the graph: including, which was the lowest bar, the highest bar and the two closest bars in terms of values.

The first study compared the multimodal approach with an exclusive haptic solution [43]. The results revealed that the WingMan mouse was not effective without audio, whereas the PHANTOM version could successfully convey the information alone. However, the multimodal condition had a shorter task completion-time and a lower perceived workload, and thus performed better overall. Hence, audio may be useful to represent the general trend and give information about the minimum and maximum values, while, in the case of value comparison, haptics was necessary. They also suggested that haptics should be used for navigation and audio to represent precise information.

The results of the second study compared the multimodal approach with traditional paper-based tactile diagrams [44]. It showed that the quantity of correct answers was greater with the multimodal system especially for the last question which utilized value comparison. The multimodal system provided better performance and accuracy than the traditional paperbased tactile diagrams but required more time and effort from the user; this was mainly because the values were realized through speech. Similar conclusions were made to previous work [31], [43], that haptics should be used for navigation and guidance tasks, while speech and non-speech audio should provide detailed value information.

In a comparison of sighted and non-sighted users, Yu and Brewster [46] noticed from the position log-file that blind users from the second study mainly used audio for information extraction and haptics for navigation, whereas the sighted users involved in the first study mainly used haptics for both tasks and audio for confirmation. This can be explained by the fact that blind users are more familiar with audio interaction methodologies through screen readers, etc. This underlines that the role of each modality depends as well on individual users.

Wall and Brewster [47] evaluating the discrimination of three haptic properties - friction, stiffness and texture - highlighted that friction gave the best discrimination results. Wall suggested mapping the value of each bar to friction, which could increase the speed and efficiency of the perception.

The same ideas were applied in the case of the pie chart

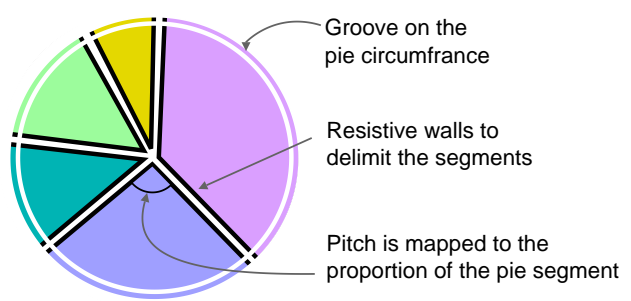

Fig. 4: The figure depicts how a pie chart is represented haptically, with resistive walls to form an enclosure for each segment, and a groove round the edge to provide an overview of the information, after [34].

by mapping the pitch to the proportion of the pie division for audio cues and by using a combination of the rectangular and the elliptic enclosure effects for haptic cues [34]. After a preliminary study, a haptic groove was added to indicate the circumference of the pie (see Figure 4) and the sound was made shorter and crisper to remove the echo. The study revealed again that the multimodal representation combining audio and haptics is more effective than either modality alone.

3) Overview and Exploration: In order to improve exploration, the concept of beacons was introduced by Wall and Brewster [48]. The concept is equivalent to making notes in the margin, and provides an external memory aid. This is a general technique that could be applied to different types of charts but was evaluated with bar-charts. A traditional qwerty keyboard was used to assign the beacons to bars, with a seek function to haptically drag the user to the beacons using an elastic spring metaphor, and audio cues were used to confirm when a beacon was placed.

Overall, most of the subjects, especially beginner users, found the beacons potentially useful, particularly for displaying complex data or to quickly move through large data sets. However, the experiment showed it was difficult to simultaneously use the keyboard and the PHANTOM. A proposed solution was to use the numeric pad to find a specific bar by pressing the corresponding number, similar to a $\mathrm{CD}$ player remote control which skips tracks avoiding the placing of beacons [48]. However this solution restricts itself to bars.

Another exploration methodology was initially presented by Wall [49] and later extended and evaluated by Wall and Brewster [50], which they named semi-passive exploration. Wall [49] proposed a dual interface approach, where the dominant hand navigates with one input-device, such as a mouse or the keyboard, and the other hand or another part of the body receives the feedback. The prototype [50] used a Wacom Intuous 2 graphics tablet augmented with tangible $\mathrm{X}$ and $\mathrm{Y}$-axis and the VTPlayer tactile mouse. The user could control the pen with their dominant hand using the tablet, with the VTPlayer mouse being used as a tactile display in the non dominant hand. The prototype was evaluated with bar-charts, where the user could feel the edges of the bars. Interviews with the five visually impaired participants provided positive feedback about the exploration usefulness and highlighted that more verbal guidance was required, in addition to the bar names and their values, as well as solid filled bars instead of the edge representation. Subsequently, the prototype was 
enhanced by filling the bars and adding contextual speech feedback (dictating whether the user was on, between or above a bar, or outside the graph) [50], but has not yet been reevaluated.

4) Automatic translation: As described in section III-A4, Yu et al. [34] developed an automatic online graph construction tool that included line graphs, bar charts and pie charts.

5) Summary: One of the challenges when developing haptic versions of charts is that traditionally developers have focused on conveying the shape of the graph, rather than representing the underlying data. Another problem arises through the limitations of the haptic device; that the device only depicts a small area of the whole representation. To overcome these inadequacies, McGookin et al. [51] proposed some guidelines. They suggested providing multiple views of haptic graphs, with each view presenting specific information through a single haptic property and answering a specific user question, while making sure that the different views are "collocating and overlapping". Lederman and Klatzky's exploratory procedures were envisaged to communicate the different views. For instance, the lateral movement associated with friction could be used to give an overview of the bar chart by attributing different frictions to each bar (see Figure 5). In addition, audio was advised to be integrated into the system to complement haptic. This idea is complimentary with the Exploded View of Roberts et al. [35], who proposed to simplify different features of the data into separate views.

\section{MAPS}

Maps communicate spatial meaning, geographic or physical space. Maps have an explicit and clear association from a point on the map to a specific point in reality. For example, a tourist map of London would show the relative positions of each of the major landmarks. Various researchers have investigated haptic visualizations that could be named maps; we sub-classify them as (1) statistical maps and (2) virtual representation of real environments. Statistical maps are referred to as 'Cartograms' in the latest classification of Lohse et al. [52], which are maps that show quantitative data.

\section{A. Statistical maps}

An example of statistical maps was developed by Jeong and Gluck [53]-[55]. They conducted several studies to evaluate the effectiveness of multimodal choropleth maps. The choropleth map enables geographical-regions to be represented by colours, where the colours realize statistical quantities of that region. The haptic display was common to each study; it consisted of different forces of vibration with periodical effect, available from the Immersion ${ }^{T M}$ plug-ins, conveyed by the force-feedback Logitech iFeel ${ }^{T M}$ mouse. Audio was used to represent different quantitative values. The first study [53], [54] looked at bivariate maps and measured completion time and recall rates to compare four models: color-color, colorauditory, color-haptic and haptic-auditory. The results revealed that the haptic-auditory display provided higher recall rates while, in regard to the completion time, the "haptic displays seem to interfere with other modalities" [53]. The second study looked at trivariate maps [55] and compared color-only and color-haptic-auditory displays. Their results showed that completion time was similar for both displays, whereas users could recall the multimodal displays better than the visual counterparts.

\section{B. Virtual representation of real environments}

Various researchers have focused on using virtual maps (primarily) to help visually impaired users learn how to navigate in a real environment. Different types of environments have been represented according to the map scale such as indoor environments, local outdoor environment (city) and global outdoor environment (country). Although the environments slightly differ, the holistic approach remains the same, which is to convey the location, size and forms of different objects and the possible paths to reach them.

1) Representation: There has been much research into the generation and use of (static) tactile maps realized on thermoform or swell paper. These static raised-maps provide an invaluable resource to those with impaired vision; such as work by Gardiner and Perkins [56] on representing tactile maps for blind golfers. However tactile map generation is out of the scope of this review. ${ }^{3}$

Schneider and Strothotte [58] presented two 2D methods where users could create haptic maps guided by the computer. First, using image processing and tracked physical bricks, users placed the bricks on a table to make the route, guided by synthetic speech commands. Second, users could follow the routes mapped out on a virtual engraving using the PHANTOM device. The usability of the haptic method was not evaluated. Parente and Bishop [59] presented the Blind Audio Tactile System (BATS) using low cost force feedback devices such as the Kensington Orbit3D ${ }^{T M}$ tactile trackball and the Logitech WingMan Rumble ${ }^{T M}$ gamepad. On a map of North Carolina users could feel subtle bumps for county boundaries, large bumps for state boundaries and a constant vibration on cities. An informal observation of four blind high school students interacting with the system highlighted they could successfully use it.

One common approach when designing haptic virtual environments is to use 3D worlds. Lahav and Modiuser [60][62] developed a 3D multisensory virtual indoor environment using the Microsoft Force Feedback Joystick. The system (see Figure 6) comprised of two modes, the developer/teacher mode enabled users to build 3D environments and lay out various haptic and audio effects, while users could navigate and receive feedback in the learning mode and be guided around objects by magnetic forces.

König et al. [63] adopted a different approach to convey architectural models. They introduced non-realistic haptic rendering: techniques that simplify the information to convey its salient features. One idea was to select a number of horizontal cuts through a 3D building, these buildingoutlines were stacked together to form a $2 \frac{1}{2} D$ structure; this

\footnotetext{
${ }^{3}$ For more information about (static) tactile maps interested readers should consult various papers [39], [40], [57] and available resources, such as the RNIB National Centre for Tactile Diagrams www.nctd.org.uk and the American Printing House For The Blind www.aph.org/.
} 


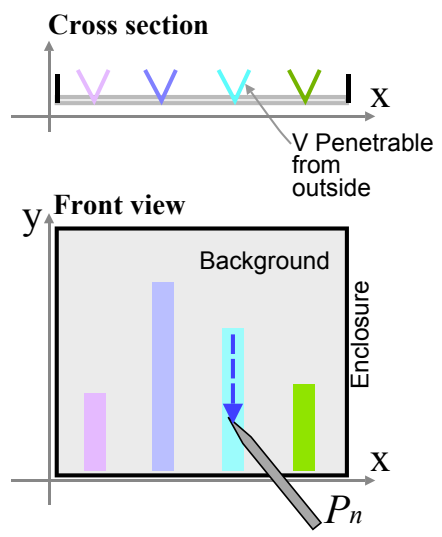

(a) Engraved bars.

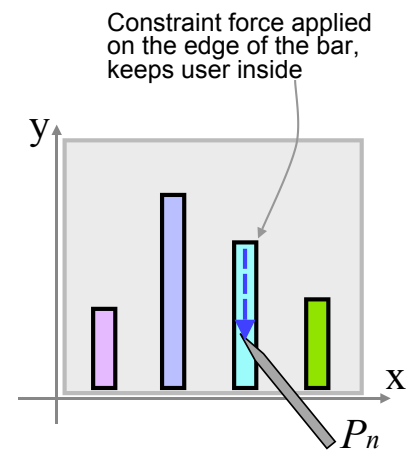

(b) Enclosures.

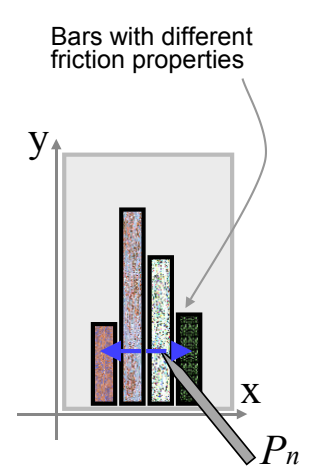

(c) Friction.

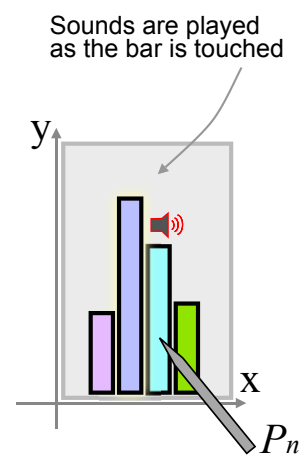

(d) Auditory information.

Fig. 5: Illustrations of different haptic bar chart techniques. 5(a) Engraved bars enable the user to explore the information [43], [44]. 5(b) Enclosures, such as created by attractive forces, have been used to keep the user on the bar [43]. 5(c) Friction can be used to distinguish the bars [51]. As the user moves horizontally he/she gains an overview of the values. 5(d) Auditory information has been used to represent the values of the bars [43], [44]

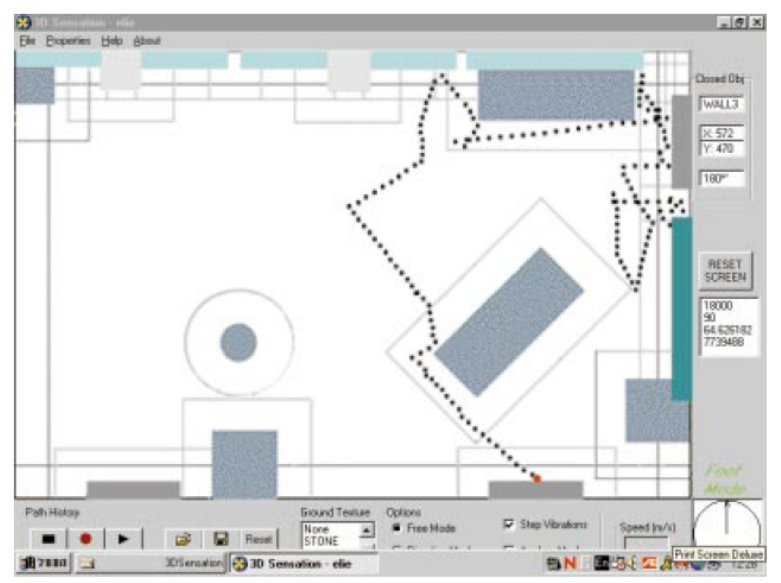

Fig. 6: The figure shows the haptic virtual environment [60] for navigating with various objects. It also shows the path of a user of the system. Reproduced with permission.

provided an understanding of the building layout but lost height information. Hence, a second model cut the building vertically, such that the user could feel the building in a scalloped form. Springsguth and Weber [64] utilize similar concepts with the structure of the map formed from the ground layer. Moreover, they assign appropriate waveforms and textures to help users distinguish objects. None of these two representation techniques have been evaluated.

All the aforementioned maps presented static data. However, Magnusson and Rasmuss-Gröhn [65] developed a complex virtual map of a real-world traffic environment presenting not only static objects but also dynamic objects of animated cars and bicycles, see Figure 7. They evaluated their tool on ten visually impaired users and found that the majority of users could navigate both the complex virtual environment and the real world scenario, and that those who could use the cane well in the real world performed well in the virtual world.

2) Exploration: Map exploration is an important task and has been the focus of various researchers' work. In fact,

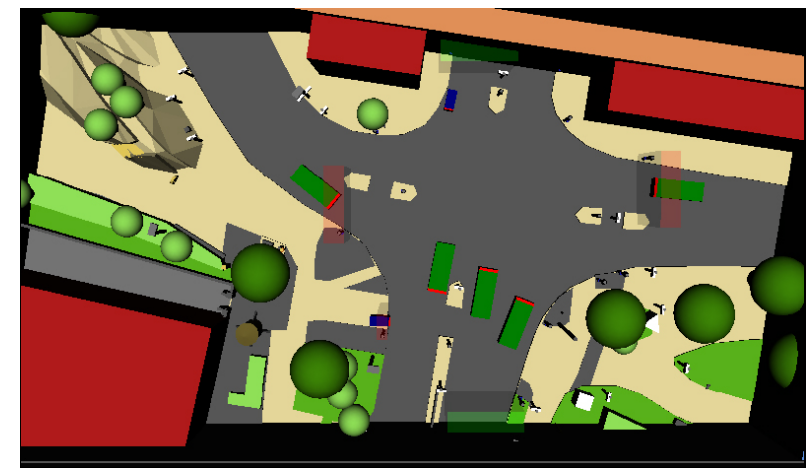

Fig. 7: The figure shows the haptic virtual model of Lund in Sweden [65]. Cars are realized by the green boxes while bicycles by blue boxes. Users could navigate using the PHANTOM and hear sounds of the environment; when hit, objects such as cars and bicycles also emitted an appropriate sound. Reproduced with permission.

different researchers have proposed a variety of techniques. Pokluda and Sochor [66], [67] categorized four exploration techniques while Roberts et al. [35] suggested others. Thus, in an attempt to categorize these exploration modalities we summarize six methods of exploration:

Free exploration enables the user to explore freely, see Figure 8(a). Many developers utilize this mode. For instance, Jansson and Pedersen [68] used the VTPlayer mouse to realize a raised version of the USA states. Lahav and Modiuser [62], using the haptic environment as shown in Figure 6, performed a study involving 31 participants to compare real and virtual exploration processes. They concluded that the haptic virtual environment was effective to enable users to develop a spatial cognitive map, which could then be transferred to the real space, and additionally it encouraged novel strategies for exploration.

Free exploration with guide permits the user to freely move around, but get additional help when required (see Figure 8(b)). For example, users can be guided to a known place when they feel lost [67], or an attraction force initiated 
when they touch an object, so as to enable them to explore that object [69], see Figure 9(b).

Free exploration with choice enables the user to move around and then be instantly teleported to another place on request (see Figure 8(c)). E.g., Magnusson and Gröhn [65] present a world where the user can freely explore in a virtual box, but when the user pushes the walls of this box, or hit a key on the keyboard, he/she is moved to another virtual world. Knowing where to explore next can be difficult; Pokluda and Sochor [67] presented a sphere-menu where the position of other objects could be indicated to the user to help in choosing the next direction to explore.

Guided tour which fully controls the movements of the user and shows them interesting features, Figure 8(d) [35], [66], [67].

Guided tour with choice, where the user is initially led and then given some choice of where to go next, Figure 8(e). Roberts et al. [35] describe a museum tour metaphor, which could be applied to maps, where the user is guided to something interesting, and then left to explore freely, before being led to something else.

Guided tour with freedom where the user is given some freedom to move as they are led along a route, Figure 8(f). For instance, Roberts et al. [35] proposed a water skier method, originally designed for line-graphs, where the user can move from side to side as they are taken on the tour. König et al. [69] presented a method where small marks (attractive forces) are placed through the scene to designate the path and guide the user, see Figure 9(c).

Pokluda and Sochor [66], [67] conducted several experiments with both sighted and blind users and obtained various results about the usability of the different exploration techniques. They concluded that all the techniques should be available to answer the individual needs and preferences.

As well as these exploration modes, König et al. [69] present active and passive, orientation and navigation strategies and provide examples with the 3DOF PHANTOM 1.5. The passive methods utilize speech to support the user, while the active methods provide forces to guide and help the user explore. For one passive feedback method, they first divide the scene into a grid then present acoustic information of the contents of the current cell. Users can then decide which cell to move onto, see Figure 9(a). While active methods provide additional forces, such as force fields, to guide the user through predefined paths or to enable users to explore specific objects. Their active classification is similar to the guided classification above. These exploration strategies have not been evaluated; however the authors report that the informal tests carried out showed promising results.

3) Qualitative information: Researchers have mostly conveyed qualitative information on the maps using the auditory modality. For example, speech has been used for objects' names and descriptions [58], [59], [61], [64] and also for directions [61], [64], [69]. Auditory icons have also been used to provide information about the objects or navigational information. Lahav and Modiuser [61] associated windows to bird chirping while Parente and Bishop [59] attributed a specific sound to each map item, such as traffic sounds for cities and bird chirping sounds for forests, emitted when near the cursor, additionally, they conveyed the direction and distance of cities through the direction and volume of the environmental sounds. Finally, Magnusson and Gröhn [65] played environmental sounds when users bumped into dynamic objects.

\section{SIGNS}

Signs are something that 'stands for' something else [70]. They may be understood because their meaning is literally denoted by itself such as being a photographic representation of an object, or implied by the environment, such as a "no right-turn' traffic sign, or merely arbitrarily assigned. Signs (semiotics) have long been studied and we refer the reader to the general semiotics literature [70] for more information. More specifically Gumtau [71] discusses haptic interfaces in the context of semiotics. Thus there are many types and forms; we categorize and introduce three levels of representation: (1) monosemic objects such as icons that have a single meaning, (2) structured monosemic objects such as earcons and tactons that again have one meaning but are structured forms, such that alike objects or actions have a similar appearance, while (3) polysemic objects are symbols that have multiple meanings such as haptic glyphs (hylphs), which are structured signs that realize multiple values, as they include multiple parts, have many ligaments and can provide quantifiable information.

\section{A. Monosemic objects - Haptic Icons and User Interfaces}

Haptic icons convey a single meaning and have a single association. They, like their visual icon counterpart, represent an idea by convention, association or resemblance. E.g., an icon on a computer interface has one program that is loaded when the icon is activated. MacLean and Enriquez write "...[haptic icons are] brief computer-generated signals displayed through force or tactile feedback to convey information such as event notification, identity, content or state" [72]. In fact, their work has resulted in the Hapticon Editor [73] and more recently extended into the Haptic icon Prototyper [74] (see Figure 10). These tools were specially developed to help design haptic icons in terms of waveforms with adjustable duration, frequency and amplitude for each waveform. The haptic icons can be created, by recording the user's 1-DOF knob motion, appending waveforms or by superposing existing icons.

In the medical domain, $\mathrm{Ng}$ et al. [75] investigated the use of haptic icons for situation-awareness in clinical operating rooms by reducing noise levels with a silent vibrotactile alarm system. Two tactors placed on the forearm were used to represent a change in a physiological signal like the heart rate. The experiment, in a simulated clinical environment, showed that the overall performance was significantly better for the vibrotactile only condition for the identification rate in comparison with an auditory-only and a combined alarm. Users mostly preferred the vibrotactile display as they found it was best method to attract their attention but the prototype did cause some discomfort.

Much of the work in the literature, in regard to haptic icons, has focused on the evaluation of the effectiveness and 


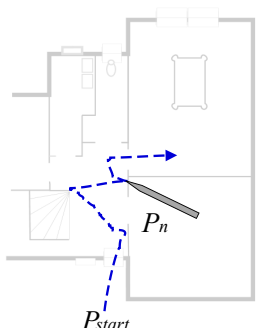

(a) Free Movement.

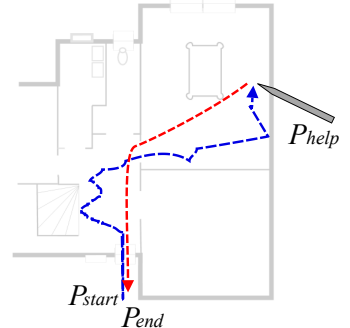

(b) Free movement with guide.

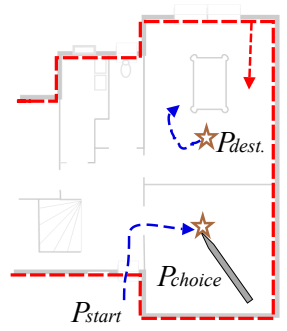

(c) Free movement with choice.

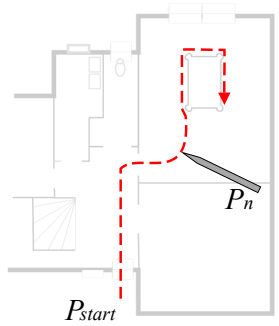

(d) Guided tour.

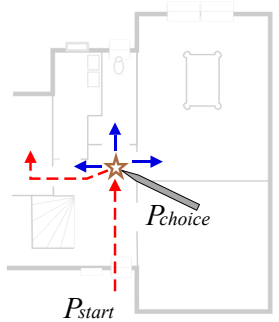

(e) Guided tour with (f) Guided tour with freechoice.

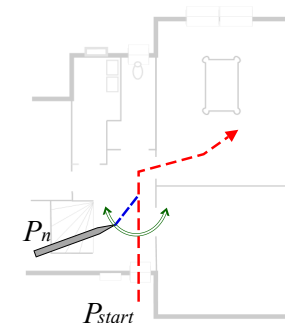
dom.

Fig. 8: There are essentially six exploration modes. 8(a) Free Movement - the user can explore anywhere. 8(b) Free movement with guide - (e.g.) if lost, the user will be taken back to a known point. 8(c) Free movement with choice - the user can move around and then choose to be hyper-jumped to another place. 8(d) Guided tour - the user is led along a predefined route. 8(e) Guided tour with choice - the user is led along a predefined route and then given a choice of directions. 8(f) Guided tour with freedom - the user is allowed some movement as they are led along a predefined route.

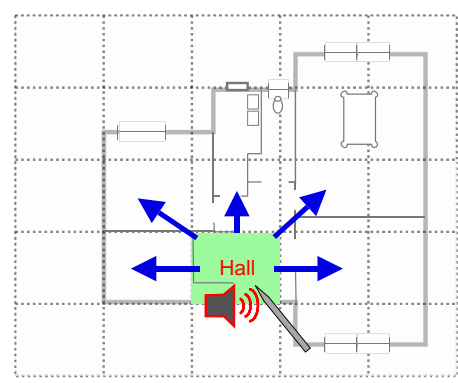

(a) Passive orientation.

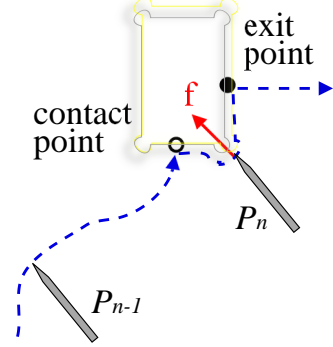

(b) Active navigation.

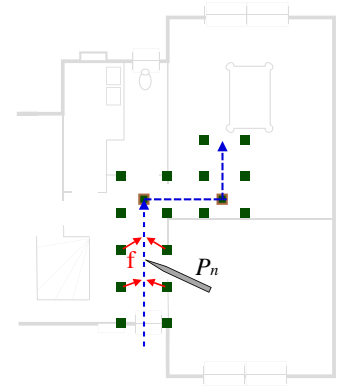

(c) Active navigation

Fig. 9: Some examples of passive and active orientation and navigation, after König, Schneider and Strothotte [69]. 9(a) Passive orientation - the user can freely move room-to-room and be led around the world through audible cues (e.g., a user heading towards a 'kitchen' may hear plate and cutlery sounds, while they may hear an alarm clock when moving towards the bedroom). The volume may increase as they get closer to the room. 9(b) Active navigation - when an object is touched a force-field will help to keep the probe on that object. 9(c) Active navigation - small marks placed through the scene designate the path; attractive forces guide the user.

discrimination of the icons. Particularly, much work has been done by Karon MacLean and her group at the University of British Columbia. MacLean and Enriquez [72] studied the perception of the wave shape (sine, square, etc.), frequency and force amplitude of haptic icons using Multidimensional Scaling (MDS). Their results showed that frequency is the dominant parameter that should be varied between 4 and $20 \mathrm{~Hz}$, followed by wave-shape and force magnitude. Chan et al. [76] evaluated the detection and identification in the case of a turn-taking collaborative environment using the Logitech iFeel mouse. Seven haptic icons were divided into three families each having a vibratory pattern (periodic vibration, buzz and taps) using frequency, magnitude, duration and the numbers of signals. They reported that haptic icons could be learned, detected and identified correctly in an acceptable amount of time under different workloads. Finally, Pasquero et al. [77] evaluated appropriate haptic properties of haptic icons, they used the Tactile Handheld Miniature Bimodal (THMB) interface with eight piezoelectric actuators. The user scenarios included list selection, scrolling, direction signalling (finding a spatial target) and background status notification. Their conclusions indicated that the haptic icons for list selection should be designed using waveform, duration and direction; for direction-signalling direction should be used, while for scrolling the parameters should be speed, direction and waveform. In a study, Enriquez et al. [78] showed that the consistent association of arbitrary meaning to haptic 'phonemes', haptic stimuli, could be learned and recalled up to 45 minutes after training.

In regard to User Interfaces various researchers have utilized haptic sensations to provide virtual feedback when a button is pressed, or to indicate other processes or commands. These are again monosemic objects as they associate one piece of information. Van Esch-Bussemakers and Cremers [79] utilized a complex setup of four PHANTOMs, with their motion restricted to a straight line, along with vibrotactile actuators integrated in the gimbals to simulate a mobile device. The setup was used to experiment how users could access a multidimensional music database. Different types of visualization (wheel, timeline, map and lexicon) could be chosen and force feedback was mainly used for navigation and selection of songs. Some vibratory patterns were implemented to convey messages such as to notify a system message or that the key/button is unavailable. An evaluation with six users showed that speech was preferred when users searched the database, with the haptic interface for selection and navigation tasks. Lee et al. [80] presented a different haptic interface. They developed a haptic pen that provided tactile feedback of 


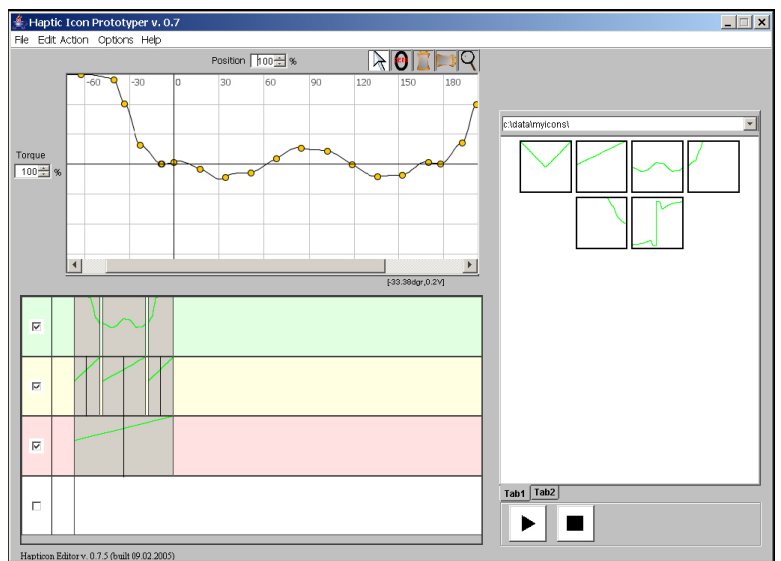

Fig. 10: Haptic icon Prototyper [74]: region (1) is the waveform editor representing the haptic signal and where the waveform can be simply changed by moving control points, region (2) is the tiles palette containing basic haptic effects and region (3) is the tile panel enabling to combine the haptic tiles. Reproduced with permission.

buttons; it used a solenoid to simulate buzzes and clicks. They mention an illustration where the strength of the buzz could change depending on the closeness to a target object. Informal usage was promising but no evaluation has been conducted. Further to this work, Lee et al. [81] used a five coin-type vibrating actuator on the fingertip, to add haptic information to a wearable and augmented reality context (AR), such that a visually impaired person could identify specific pictograms or signs. However the system has not been evaluated.

There are a few challenges to overcome when using haptics in interfaces, especially haptic icons and buttons. First, the user needs to search for the information [82], for instance, Sjöström [83] proposed a simple cross hair cursor as a solution to enable the user to scan the $\mathrm{X}$ and/or Y-axis for the target icons. Second, the user needs to discern that objects are distinct. For instance, Lee and Hannaford [84] experimented on "haptic thresholds of the index finger ... for two icon alignments" discovering that the thresholds ranged from 15 to 24 milliNewtons, and that finger motion would affect the threshold.

\section{B. Monosemic objects with structure - Tactons}

Drawing inspiration from the visual (icons) and especially the audio domain (earcons), tactile icons named tactons were designed by Brewster and Brown [85]. Vibrotactile devices were used to generate the signal, while the information was encoded in using frequency, waveform, amplitude, duration, rhythm and spatiotemporal patterns. Three types of tactons have been proposed: compound tactons, hierarchical tactons and transformational tactons. Compound tactons aim to create a language. For example a gradually-increasing high-frequency pulse could represent loading a file, while a similar, but dual frequency vibration could represent saving a file. Hierarchical tactons are tactons that can inherit properties from their parents in a tacton tree. Finally, transformational tactons have several properties each mapped to a different tactile parameter. For instance, a file contains attributes such as size, location, date, which could be mapped onto frequency, body location and rhythm, respectively. Transformational tactons are in fact an example of polysemic objects, and are thus included in the polysemic subsection $\mathrm{V}-\mathrm{C}$. Tacton research suggests that rhythm and spatial location were effective parameters for tactons, while the vibrotactile roughness (created using amplitude modulation) was not effective. Consequently, Hoggan and Brewster [86] conducted a study to find new efficient techniques to represent tactile texture and they concluded that using different waveforms was the best technique (recognition rate of $94.2 \%)$.

1) Compound tactons: Compound tactons are often made up of multiple actuators; they can be used to convey geometric information, warning signals, and other coded information [87]. These tactons can be useful in situations where sound, visuals or other senses are already overloaded. E.g., Van Veen [87] present an experiment where they proved that under high G-load conditions, vibrotactile stimulation with a torso display is not degraded whereas the visual channel does degrade or become unusable.

Compound tactons have been applied in various domains. Brown et al. [88] used vibrotactile tactons to encode different types of phone-message call (voice call, text message, or multimedia message). The Engineering Acoustics C2 Tactor $^{T M}$ vibrotactile device was used with modulated sine waves to represent the call types, and the roughness the priority (low, medium or high). They highlighted that rhythm was very successful (with a 93\% recognition rate) and that roughness was usable (with $80 \%$ recognition rate). Such vibrations have been long used in clocks to tell the time. Töyssy et al. [89] encoded the number ten with long pulses and one with short pulses with a break of two second between the hour and the minute parts (i.e. L-L-S-S-S means 23 and L-S means 11, giving 23:11). After conducting two experiments, they concluded that this mapping was viable and easy to learn. Other researchers have used multiple actuators to encode directions; developing tactile vests, suits, chairs or belts to encode direction.

Ertan et al. [90] designed a wearable haptic vest including a 4-by-4 stimulator array to help the blind and visually impaired people navigate the world. Five vibratory instructions (four directions and stop) could be transmitted through the array using patterns. Although there were some problems with the position sensing system, the users gave good feedback. For a similar application, Bosman et al. [91] presented a wearable wrist-band device that enabled sighted users to navigate real environments through vibratory directional instructions. An experiment conducted with 16 participants highlighted that the system was helpful and "a practical way to deliver guidance information for pedestrians indoors", reducing the number of errors to reach the destination as compared with signage. Similarly, Jansen et al. [92] designed a tactile shirt display (a horizontal tactor belt and a vertical part on the back), to provide helicopter pilots with quantitative information about altitude and groundspeed when landing in degraded visual conditions also referred to as 'brownout' conditions. They conducted a functional test that highlighted that landing performance in degraded visual environments was significantly improved with the tactile display. 
Rupert [93] proposed the Tactile Situation Awareness System (TSAS), a torso suit containing an array of multiple electromechanical tactors and a wearable computer to prevent spatial disorientation in aeronautical or unusual acceleration environments. The TSAS was used to provide information about pitch and roll for aircraft simulation for instance or airspeed or velocity in the case of helicopter flights. Overall, users understood the information and achieved good manoeuvres performance; however the limitations of the tactors and the suit did not provide fully effective and comfortable transmission of tactile cues.

Van Erp and van Veen [94] designed an in-car tactile navigation display with tactile actuators mounted in the seat in order to solve visual distraction or workload. A feasibility study evaluated the reaction to messages about a course change where direction was encoded by the location and motion properties and distance by rhythm. Measurements of workload, mental effort and performance under visual only, tactile only and visual+tactile conditions stressed that a vibrotactile display in cars is useful to improve efficiency and safety [94] as the tactile only condition achieved the best results. Van Erp et al. [95] followed their work by evaluating a vibrotactile belt with eight tactors delivering direction indications to a helicopter and a fast boat pilots. The tactile display proved that it can be successfully and easily used in waypoint navigation and even in vibrating environments. In a similar application Tan et al. [96] developed a haptic back display which used a 3-by-3 tactor array and also proved that such a display was effective for haptic attentional and directional cueing.

One of the challenges with vibrotactile jackets is that they are tethered to a computer. Recently, Lindeman et al. [97] have conducted extensive research into wearable vibrotactile displays with the goal of producing a highly reconfigurable, unencumbered, full-body haptic wearable system. They have developed the TactaBox, which is a wireless box that controls 16 tactors through lightweight cables; TactaVest and the TactaPack (wireless tactor units). Their testing included using the belt from the TactaVest along with the Tactabox, with eight-tactors, in a building-clearing task with 28 subjects. An experiment was conducted evaluating the task with and without the vibrotactile cues. The vibrotactile system allowed the user to spend significantly less time clearing a larger percentage of the space [97]. Jones et al. [98] also developed a wirelessly controlled tactile display, comprising a 4-by-4 array of vibrating motors for the lower back. This system was displaying tactile patterns tested for navigation in both laboratory and outdoor settings. The experiments indicate that vibrotactile cues or tactons presented to the torso could be accurately and easily identified, reaching 100\% recognition rates for most patterns.

2) Hierarchical tactons: An example of a hierarchical tacton is described by Osawa [99] (although he named them tactile glyphs). He utilized the vibrotactile stimulators of the CyberTouch $^{4}$ glove to represent nodes of a hierarchy. Different stimulators on the fingers or palm were activated in turn

\footnotetext{
${ }^{4}$ CyberTouch ${ }^{T M}$ is the vibrotactile feedback option for Immersion's CyberGlove glove. See www.immersion.com
}

to encode the position of that node in the hierarchy. Two experiments with 42 and 26 users respectively highlighted that it is better to use presence of tactile stimulus rather than strength variations as well as a small number of stimuli to improve the accuracy. These experiments intended to find the right parameters to construct discriminable 'tactile glyphs', thus no evaluation within a context of use has been conducted at that stage.

Recently, various researchers have shifted their focus to mobile applications. For example, Hoggan et al. [100], [101] used multiple actuators to provide information about a progress bar and feedback while entering text on a handheld device. By using spatial location and rhythm for the tactons, their study highlighted that tactile information reduces the error and increases performance as compared to visual interfaces. Many of these systems are 'interfaces' rather than visualizations, because they realize a single value rather than providing any quantifiable information. Thus we refer the reader to the related areas of wearable computing [102]-[105], human computer interaction (HCI) [106] and virtual reality [5], [11] for additional information on mobile applications.

\section{Polysemic objects - haptic glyphs, transformational tactons and hlyphs}

Glyphs in graphical visualization are structures that realize multiple values: they may change their shape, form or size, either in full or in part to realize different values and information. They are certainly similar to tactons. However, tactons (excluding transformational tactons) are used to map an identity (e.g., up, down, left or right), whereas haptic glyphs (or hlyphs) focus on describing quantitative information (e.g., move forward by 30 miles-per-hour).

A good example of a haptic glyph is by Dobson et al. [107] who introduce an interface called 'what's shaking'. As the user navigates newsgroups so the amount of postings from individuals is mapped to vibration of a tactile glove and temperature of that glove, such that people perceive "populous groups as warm and active groups as vibrant". An informal test with 12 students showed that they were able to get an understanding of a newsgroup social activity through the use of temperature and vibration. Another example is the electronic cane of Hoyle et al. [108], where vibration increased as the user approached an object. Extensive trials with 27 visually impaired volunteers and other reviewers and advisors led to positive comments about the usefulness of the cane.

Brewster and King [109] compared a transformational tacton version of a progression bar with a visual progression bar. They used the Tactaid VBW32 ${ }^{T M}$ tactile transducer, and the time remaining was represented by the amount of time separating two vibrations. The vibrotactile progression bar enabled a reduction in time to notice the end of the task of $36 \%$ in comparison with the visual progression bar.

Furthermore, Roberts and Franklin [110] name the haptic glyphs "hlyphs" and present some design guidelines to enable easy navigation and exploration for (in particular) forcefeedback hlyphs. They should be (1) well structured, (2) compound and multifaceted such that multiple values can 
be represented, (3) self contained such that the user does not confuse multiple glyphs, (4) endogenous in design as it is easier to navigate a force-feedback device (such as the PHANTOM) internally, e.g. a valley is easier to navigate than a ridge [31], (5) enable pre-attentive perception such that the user implicitly understands the data, (6) utilize conceptual mappings to implicitly understand the mapping function that has been used, and (7) have an appropriate affordance such that the user implicitly knows how to operate it.

\section{NETWORKS}

Networks describe relational information, including trees, hierarchies and paths, e.g. $a$ connects to $b$ then $c$. A good example of a hierarchy is blood vessels. Yi and Hayward [111] used the 3D force-feedback PenCat/Pro ${ }^{T M}$ interface to enable users to haptically navigate through blood vessels from volume angiograms. The user could see a $2 \mathrm{D}$ volumetric projection of the $3 \mathrm{D}$ vessel network and zoom in and out of the network. The haptic feedback was used to provide depth information; the harder the user pushes, the larger the forces are. A preliminary study with four students including the first author exhibited a mean accuracy of $99.2 \%$ and a mean decisionmaking time equal to 5.4 seconds when discriminating the depth relationship between two separate dots on the cerebral vessel.

Typical network visualizations focus on displaying the relationships among components [52]. Thus, to understand a network a user must identify each component or node in comparison to its neighbours, and perceive the holistic structure of the chart. Improving the haptic visualization of network charts therefore implies improving these two tasks. In that respect, Jay et al. [112] developed some guidance interactions to improve the "recognition of abstract data at both the micro (individual nodes) and macro (overall structure) level". They used a spring force to guide the user to the leftmost node and a force constraint to keep the user on the surface of the object while he/she explores it. Subsequent nodes could be investigated by pressing the space bar, which moved the spring onto the next node. Audio cues, which were played on demand, indicated the node type: a wind-chime sound identified a sphere while a bicycle bell represented a cube, the two objects used in the experiment. Nine blindfolded (sighted) participants were evaluated under four conditions: namely no cues, audio cues only, haptic cues only and both audio and haptic cues. This was done to evaluate the impact of haptic cues on both the identification of nodes and whether users understood the overall structure. The results showed that the multimodal cues provided the best results, leading to faster recognition of both nodes and structures and more accurate recognition of nodes (100\% of correct answers) than in the other conditions. All the participants rated the audio cues as useful for recognizing nodes, and haptic cues as useful for recognizing structure, which validates the design of the role of each cues. This work highlights that constraining the user haptically can help the user understand the structure. Potentially these ideas could be applied to other areas such as Diagrams, Charts and Maps.
Another form of relational and progressive information is music. Although not a dynamic tactile display, Challis and Edwards [113] presented a touchpad with tactile overlays to control. Vacuum formed overlays were used to represent parts of the score, which could be used to control the music. E.g. touching on a bar line would play the music of that bar. An observational study with six users led to constructive comments to improve the interface (such as avoiding empty space and the double click was difficult to achieve without haptic feedback). Chu [114], on the other hand, designed the TouchSound software: a digital sound editing software with haptic feedback to enhance the interaction and especially the efficiency; but did not conduct a user study to evaluate it. Haptic feedback was used to enable users to feel and locate features in the sound such as beats, and users could feel short periodic oscillations or strikes when they were strong. A potential group of users of such an application could be DJs [114] as they need to know the features of the music record to mix it with another song. In fact, Beamish et al. [115], [116] developed the D'Groove, a haptic turntable for DJs to enable them to use digital format without losing the feel and advantages of the traditional setup. The DJ could feel the beat structure through virtual bumps at every beat, musical events through friction proportional to amplitude, the turntable platter or the Q-slider (that replaces the needle) is harder to move in frequency rich moments and inversely and could create new effects with springs and texture. The observational study with six experienced DJs [116] resulted in an overall positive feedback and highlighted the need for a self-contained turntable unit with higher turntable torque and better appearance.

\section{DIAGRAMS}

Diagrams illustrate some process, phenomenon, or concept. In visual terms they include schematic diagrams and illustrations. For instance, a diagram may be used to illustrate the process of constructing a flat-pack cupboard, or could be used in a botanical encyclopedia to illustrate and label the leaf types of different trees. Lohse et al. [52] distinguish two types of diagrams: structure diagrams and process diagrams. Structure diagrams are "a static description of the physical object. The spatial data expresses the true coordinate dimensions of the object." while the process diagrams "describe the interrelationships and processes associated with physical objects. The spatial data expresses dynamic, continuous, or temporal relationships among the objects in process diagrams". No work, as known to the authors, has been done on structure diagrams. However, the work done in scientific visualization can be classified as a combination of a structure and process diagrams, as haptics is often used to provide more insight into the physical structure of the objects along with the processes associated with these objects.

\section{A. Scientific visualization diagrams}

Taylor II et al. [117], [118] used force feedback with a Nanomanipulator for the manipulation of surfaces at nanoscale. This atomic scale teleoperation enabled the users to learn 
about the surface properties, to more effectively explore it and to interactively modify the surface with near real-time observations of dynamic processes [117], [118]. Through collaboration with scientists, force feedback control was found to be useful as it enabled finer control and touch; facilitating users to locate the correct point and the right modification path [118], [119]. Various researchers have created molecule docking visualizations. Brooks, in the GROPE project [119], [120], presented a molecule docking example where users could view molecules, interact and feel the different forces from individual molecules. The experiment, conducted with 12 experienced biochemists, showed that $6 \mathrm{D}$ rigid body docking manoeuvres were performed faster and that the chemists proceeded more directly toward the correct minimum when using haptics as compared to a visual-only display [120]. Most importantly, the chemists reported getting better understanding of the situation and the problem. Sankaranarayanan et al. [121] developed a similar haptic display, although not evaluated, to accelerate the learning process in molecular biology. Using the PHANTOM, users could feel the forces of the molecules and view them in augmented reality. Similarly, Durbeck et al. [122] used a visual and haptic display to represent vector fields. The display could represent various fields including gravity, pressure, force, current, velocity and scalar gradients, and that could be felt with a PHANTOM. No evaluation has been reported. Finally, Wies et al. [123] used speech and force feedback with the Logitech WingMan Force Feedback mouse to let students find the charge on an uniformly charged sphere in the education of physics and especially electric fields for blind and visually impaired students. A feasibility study conducted with four blind students and educational experts led to very positive feedback about the usefulness of haptics and the system for accessible education.

Haptic volumetric field visualization has been researched by a few research groups. First, Avila and Sobierajski [124] used the PHANTOM to convey volumetric information. They detailed both an isosurface and volume rendering of the volumetric data. Their isosurface was calculated as a solid surface, whereas the volume rendering mimicked the gel-like representation of its graphical counterpart, with more opaque material being realized by a greater motion restraint. The system was not evaluated but the authors found "that the integration of haptic interaction into a scientific visualization process can lead to a better understanding of complex, threedimensional data, and can result in more natural, intuitive methods for modifying this data". Second, Lawrence and Pao [125], [126] and their colleagues described various haptic flow-visualization applications. They presented various rendering modes for scientific visualization including those for vector and tensor fields, "which are types of data that are difficult to graphically visualize" [125]. For vector fields these included orientation constraint that constrains the users' stylus to the direction of the vector, transverse damping where forces are applied "in directions transverse to the field .. and forces proportional to the field magnitude", and relative drag where forces are applied proportional to the "difference between vector fields values and the user's hand". They have presented various applications of these ideas including a shock and vortex visualization [127] and work on combined visual/haptic rendering modes [128], which detailed various techniques including a flow field visualization where the orientation of the haptic actuator was constrained to depict the orientation of the flow, and a constraint method to enable the user to follow the isolines of the flow in a vortex visualization. Although the authors report the benefits of augmenting graphical visualizations with haptics to convey the information, no formal user evaluation testing the usefulness of the rendering modes has been conducted. Reimersdahl et al. [129] extended Pao et and Lawrence's work [125] and presented various haptic rendering techniques for the interactive exploration of Computational Fluid Dynamics (CFD) datasets in virtual environments. After an initial evaluation with four participants, they concluded that adding haptics was "a very promising approach to facilitate the exploration process of CFD simulation data". Mendez et al. [130] developed two haptic guidance navigation methods to help users locate regions of interest in volumetric data. They provide a 2D solution for the exploration of 3D datasets, by using the Proactive Desk haptic feedback device, which solves the common problem of occlusion in $3 \mathrm{D}$ representations, but they have not evaluated it. Lundin et al. [131] have developed 'haptic primitives' (directed force, point, line, plane) and 'haptic modes', which are high-level haptic interactions definitions (such as viscosity, gradient force, vortex tube) based on the haptic primitives. They have integrated these modes into the Volume Haptics Toolkit (VHTK). A pilot qualitative study was conducted with seven experienced radiologists with the task of exploring the blood flow in the heart and identifying paths using stream ribbons and some haptic modes. It confirmed that adding force feedback was useful for information and guidance and contributed to the understanding of the distributed flow; they concluded that "haptic feedback was considered helpful and the combination of haptics/graphics produced a better result than using visual feedback alone" [131]. Recently, Palmerius and Forsell [132] also tested three different haptic metaphors (line, surface and force) representing volumetric vector data to identify faint structures. The experiment conducted with 12 participants showed that the line and the surface metaphors permit the user to better identify faint structures in comparison to the force metaphor.

\section{B. Other diagrams}

Various other diagram styles exist. For instance, a block diagram is a simple abstract diagram of a process or system. Kahol et al. [133] treated block diagrams as images and hence used computer vision techniques to parse and then convey the information through audio and haptics. Speech was used to narrate the features and tactile feedback, via a custom made glove, indicated the presence of block diagrams components and their position: long pulses indicated text, with $(\mathrm{x}, \mathrm{y})$ coordinates being mapped to the duration of a tactile pulse. They report that an initial user study led to a $100 \%$ recognition accuracy for the shapes of the blocks and that users commented on the tactile feedback as being very helpful. Likewise Horstmann et al. [134] developed a system to 
automatically translate technical diagrams into tactile versions. The user could navigate the information through the keyboard and force-feedback joystick. However, the haptic mapping used is not very detailed and thus it is unclear whether the haptic feedback contributed to the reported users' ability to build up a coherent representation of the diagrams during the evaluation. We classify this work ( [133], [134]) under Diagrams but specific use cases could also fit in Networks. For instance, Horstmann et al. [134] applied their system in three domains: "analogue and digital electronic circuits, certain UML [...] diagrams and architectural floor plans". The architectural floor plans could be classified in the Networks section while the circuits and UML diagrams would classify more as process diagrams as they tend to focus not only on the elements' relationships but also the processes involved. On the other hand, block diagrams can either focus more on elements' interconnections and relationships or on processes, therefore suitable both for the Diagrams or Networks sections.

Finally, researchers have developed various ways to convert semantic web, or other structured diagrams into non-visual forms. Although these are not necessarily examples of dynamic visualization they are highly relevant to this section. First, Lorenz and Horstmann [135] present a short paper on the 'semantic access to graphical resources for blind users'. Fredj and Duce [136] present GraSSML which utilizes Scalable Vector Graphics (SVG) to develop a high-level description language that can be applied to different modalities. Lastly, Krufka and Barner [137] present some technical advice on how to automatically convert SVG into tactile graphics, which were printed to paper using a Braille printer.

\section{IMAGES \& PHOTO-REALISTIC RENDERINGS}

Images and Photo-realistic Renderings provide realistic representations of real world objects or scenes. Way and Barner [39], [40] in 1997 presented two seminal papers on the automatic translation of images into a tactile form, felt with the microcapsule paper. Furthermore, and in the same year, Kurze [138] presented a haptic renderer that converts a 3D scene into a $2 \mathrm{D}$ form that can be printed as a raised line drawing or used in some systems for interactive exploration [139]. Although these are foremostly static visualizations they are important and relevant to this paper because they utilize aggregation and simplification methods that are necessary for haptic visualization. Additionally, Kurze [139] developed a navigation aid (TGuide) to interactively explore tactile images [138]. The system utilized a custom made mouse with eight vibrators, a digitizer pen and a speech recognition and synthesis system. Their custom device could be used in a twohanded mode with one hand resting on the device, or in a one-hand mode with the custom device and pen together. He proposed two guidance modes: a tourist metaphor where the user is guided from one object to the next (the guided tour with choice design, see Figure 8(e)) and a scout metaphor where the user can ask for the location of an object through simple spoken commands (the free movement with guide design, see Figure $8(\mathrm{~b})$ ). The guidance was provided through directional hints by activating the corresponding vibrator. An evaluation was conducted with 12 sighted and blind people for a guidance to a target task and compared a visual, an acoustic and a tactile condition. It highlighted that the tactile directional guidance was more efficient than acoustic guidance, and comparable to visual guidance and was judged of "great value" by the blind users.

Various other researchers have developed haptic renderings to realize three-dimensional buildings and images. For example, Pokluda and Sochor [67] used the PHANTOM to enable users to explore or be led around a building, and Laycock et al. [140] permit users to navigate a high quality rendering of an ancient hall using a PHANTOM Omni. Whether the generation of high-quality renderings is useful is a matter for discussion, for instance, König et al. [63] discuss that users find it difficult to perceive shapes, sizes and complex objects effectively and that abstract (non-realistic) renderings are more beneficial.

\section{TABLES}

Tables display information in a tabular form. I.e. the data is structured in a grid format. Different kind of information can be depicted in a grid format, including numerical (such as spreadsheet layout) or graphical (such as the reorderable matrix [27], [141]), or sonified [142]. In information visualization tabular representations are extremely popular. They display multidimensional data and enable the user to compare values and perform correlations. Various researchers have looked at sonified tables (e.g. [142]), and Kildal and Brewster [143] used tactile feedback to enhance their sonified table (TableViz), but to date no haptic visualizations could be classified as representing tabular data. On the one hand, current haptic devices do not lend themselves to tabular representations. This may be because many of these devices are point-based devices, which make it difficult to convey exact values haptically. While on the other hand, specific modalities may be better at displaying certain data; and be most relevant to a particular task. For instance, van Esch-Bussemakers and Cremers [79] investigated the use of different modalities to operate different functions; concluding "that participants did have preferences for certain modalities when handling certain types of information".

\section{Discussion}

The classification of the various papers is based on deep reflection and research; however some of the papers could be placed under several categories. E.g., Yi and Hayward [111] present a method to haptically navigate through blood vessels, and thus we categorized it as a network technique, but it could be classified as being a diagram representation; their example demonstrates a hierarchical form which we believe is better described in the Network category. Another challenge is to separate Networks from Diagrams. In particular this affects the work on haptic music. Music could be listed as a Diagram as it demonstrates a process. However, we believe music fits better in Networks (see section VI) because it demonstrates many structured relations which could be hierarchical. This concurs with the classification by Lohse et al. [52] who included music under Networks. 
The classification of the papers is perhaps however a minor challenge; a greater challenge is how the user and reader can generate appropriate haptic visualizations. In fact, multiple challenges exist. Developers need to choose appropriate mappings (based on how the user will perceive that information), develop effective models, provide efficient interaction techniques, and utilize the most appropriate hardware.

Currently there are few haptic visualization guidelines to help create effective haptic visualizations and they are scattered among the publications. Thus, there is a need to produce and gather effective guidelines for haptic data visualization. Some guidelines do exist though in the more general context of designing haptic interactions and they are currently being collated under the ISO Standard for tactile and haptic interactions [144]. Following from guidelines, evaluation is an important aspect of any haptic presentation development. We have described some evaluation studies but certainly more are required.

We believe that effective haptic data visualizations should be using haptic properties both at the representation level (e.g., engraved lines, bumps) and at the task level (navigation, selection, manipulation). However, it would seem that many researchers have focused on designing haptic representations that are similar to their visual counterparts rather than finding the best haptic visualization for that task. Although it may be useful to learn from the visual domain it is better to develop specific visualizations [145] and developers should think carefully how they can best display their information through haptics. Researchers have started to think about the effective design issue [36], [51], [146] but more work is required.

Navigation and exploration tasks are another important and timely challenge. It is often difficult for users to understand where they are located. With visual information users gain an overview by scanning their eyes over the whole display. Haptically scanning over the whole display is difficult and often impractical. Franklin and Roberts [36], [110] describe various guided-tour methods to aid the user to haptically understand the context, while Panëels et al. [38] demonstrate a toolkit to create various haptic navigation methods. Other researchers have utilized additional modalities to give the user an understanding of context, place and value, often relying on speech and sonification (E.g. [33]). Palmerius [132] writes on haptic interaction design saying that it is useful to "not only design the feedback to most effectively convey the information, but to convey it in an intuitive manner so not to confuse the user". In particular choosing the right hardware (haptic device) is also important and can affect the users' perception of the information.

Indeed, we have mentioned in the classification the specific haptic device used by the designer, because in addition to the importance of the type of technology used (tactile or force feedback), each device adds its own limitations and challenges (i.e. mouse vs stylus interactions, 2D/3D). The wide use of vibrotactile tactors and point-based force-feedback devices demonstrates the usefulness of these devices, however, they may not be suitable for the task, and thus researchers should be encouraged to find the right haptic device for the task, rather than merely reaching for the most available one. Researchers should also try to compensate for the specific limitations of the device through the use of new interaction metaphors (passive/active exploration).

Generally, the haptic representation and interaction techniques for the tasks are determined on a case-by-case basis (or at least within a category such as Charts, Maps, etc.); however ideas for representations and tasks (valleys instead of ridges and exploration techniques) can be reused across applications and research areas and should be therefore investigated.

\section{CONCLUSION}

This article presents the state of the art in Haptic Data Visualization. The scope of the work presented here has been towards data presentation using haptic devices. Two areas that would expand the paper substantially are to include the haptic papers from Virtual Reality and Medical Applications. But we deemed these subjects to be outside of the scope of this paper because they tend to focus on user interface methodologies and represent 'state' rather than 'value'.

By the very nature of this review presentation readers can easily view where researchers have focused their effort: that most research has been to perform charts, in particular many researchers have focused on line graphs, bar charts and pie charts. Three further areas that researchers have investigated are maps, diagrams and signs. Researchers have looked at virtual representations of real environments, scientific 'diagrams' (such as molecular docking and field flow), and signs particularly monosemic objects of haptic icons, user interfaces and tactons. However, significant lack of development has been in the areas of networks, images and photo-realistic renderings and tables.

Finally, we believe that the area of haptic visualization will continue to grow and develop, helped along by research in perception especially perception and interference of multimodal solutions, aided by new devices and technologies and inspired by ever complex information visualization solutions.

\section{APPENDIX \\ SUMMARY OF REFERENCES}

This appendix provides the references classified according to the section they are cited.

\begin{tabular}{|c|c|}
\hline Introduction & [1] - [11] \\
\hline Background & $\begin{array}{l}\text { HDV model [4], [12] - [23]; Classification [4] - [6], [24] } \\
-[28]\end{array}$ \\
\hline Charts & $\begin{array}{l}\text { Line charts [2], [8], [29] - [41]; Bar and Pie Charts [30], } \\
{[31],[33]-[35],[42]-[51]}\end{array}$ \\
\hline Maps & $\begin{array}{l}\text { [52]; Statistical maps [53] - [55]; Virtual Representation } \\
\text { of real environments [35], [39]- [40], [56] - [69]; }\end{array}$ \\
\hline Signs & $\begin{array}{l}{[70],[71] \text {; Monosemic objects }[72]-[84] \text {; Monosemic }} \\
\text { objects with structure }[5],[11],[85]-[106] \text {; Polysemic } \\
\text { objects [31], [107] - }[110]\end{array}$ \\
\hline Networks & {$[52],[111]-[116]$} \\
\hline Diagrams & $\begin{array}{l}\text { [52]; Scientific visualization diagrams [117] - [132]; Other } \\
\text { diagrams [133] - [137]; }\end{array}$ \\
\hline Images & {$[39],[40],[63],[67],[138]-[140]$} \\
\hline Tables & [27], [79], [141] - [143] \\
\hline Discussion & {$[33],[36],[38],[51],[52],[110],[111],[144]-[146]$} \\
\hline
\end{tabular}




\section{REFERENCES}

[1] R. B. Loftin, "Multisensory perception: Beyond the visual in visualization," IEEE Comput. Sci. Eng., vol. 5, no. 4, pp. 56-58, Jul/Aug 2003.

[2] J. C. Roberts, "Visualization display models - ways to classify visual representations," Int. J. Comput. Integrated Des. Construct., pp. 1-10, Dec 2000.

[3] C. B. Zilles and J. K. Salisbury, "A constraint based god-object method for haptic display," in IROS '95, vol. 3. IEEE Computer Society, 1995, pp. $146-151$.

[4] M. Benali-Khoudja, M. Hafez, J.-M. Alexandre, and A. Kheddar, "Tactile interfaces: a state-of-the-art survey," in ISR'04, Paris, 2004, pp. $721-726$.

[5] R. J. Stone, "Haptic feedback: A potted history, from telepresence to virtual reality," in Workshop on Haptic Human-Computer Interaction, ser. LNCS, S. Brewster and R. Murray-Smith, Eds., vol. 2058. Glasgow, UK: Springer-Verlag Berlin Heidelberg, 2000, pp. 1-7.

[6] V. Lévesque, "Blindness, technology and haptics," Haptics Laboratory, Centre for Intelligent Machines, McGill University, Montréal, Québec, Canada, Tech. Rep. TR-CIM-05.08, Aug 2005.

[7] C. C. Chase, P. J. Chenoweth, R. E. Larsen, T. A. Olson, A. C. Hammond, M. A. Menchaca, R. D. Randel, M. A. Srinivasan, and C. Basdogan, "Haptics in virtual environments: Taxonomy, research status, and challenges," J. Computers and Graphics, vol. 21, no. 4, pp. 393-404, July 1997.

[8] J. P. Fritz and K. E. Barner, "Design of a haptic data visualization system for people with visual impairments," IEEE Trans. Rehab. Eng., vol. 7, no. 3, pp. 372-384, Sept 1999.

[9] K. Salisbury, F. Conti, and F. Barbagli, "Haptic rendering: Introductory concepts.” IEEE Comput. Graph. Appl., vol. 24, no. 2, pp. 24-32, 2004.

[10] C. Basdogan, S. De, J. Kim, M. Muniyandi, H. Kim, and M. A Srinivasan, "Haptics in minimally invasive surgical simulation and training." J. Comput. Graph. Appl., vol. 24, no. 2, pp. 56-64, 2004.

[11] C. Youngblut, R. E. Johnson, S. H. Nash, R. A. Wienclaw, and C. A. Will, "Review of virtual environment interface technology," Institue for Defense Analyses (IDA), Tech. Rep. IDA Paper P-3186, March 1996.

[12] R. B. Haber and D. A. McNabb, "Visualization idioms: a conceptual model for scientific visualization systems," in Visualization in Scientific Computing. IEEE Computer Society Press, 1990, pp. 74-93.

[13] C. Basdogan and M. A. Srinivasan, "Haptic rendering in virtual environments," in Handbook of Virtual Environments: Design, Implementation, and Applications. LEA, 2001, ch. 56, pp. 117-134.

[14] M. C. Lin and M. A. Otaduy, Eds., Haptic Rendering - Foundations, Algorithms, and Applications. AK Peters, 2008.

[15] M. Lin and S. Gottschalk, "Collision detection between geometric models: a survey," in IMA Conference on Mathematics of Surfaces, 1998, pp. 602-608.

[16] T. G. Zimmerman, J. Lanier, C. Blanchard, S. Bryson, and Y. Harvill, "A hand gesture interface device," in ACM CHI and GI. ACM Press, 1987, pp. $189-192$.

[17] D. L. Peterson, "Computer-controlled tactile display," Master's thesis, Department of Electronic Engineering, MIT, 1967.

[18] "Haptics photo gallery," The Haptic Community Website. [Online]. Available: http://haptic.mech.northwestern.edu/intro/gallery/index.html

[19] V. Hayward, O. R. Astley, M. Cruz-Hernandez, D. Grant, and G. Robles-De-La-Torre, "Haptic interfaces and devices," Sensor Review, vol. 24, no. 1, pp. 16-29, Feb 2004.

[20] G. Burdea, E. Roskos, D. Silver, F. Thibaud, and R. Wolpov, "A distributed virtual environment with dextrous force feedback," in Intl. Conf. Interface to Real and Virtual Worlds, 1992, pp. 255-265.

[21] G. C. Burdea and N. A. Langrana, "Virtual force feedback: Lessons, challenges, future applications," IEEE/ASME Trans. Mechatron., vol. 5, no. 2, pp. 178-182, 1993.

[22] G. Burdea, J. Zhuang, E. Roskos, D. Silver, and N. Langrana, "A portable dextrous master with force feedback," Presence, vol. 1, no. 1, 1992.

[23] H. Iwata, "Artificial reality with force-feedback: Development of desktop virtual space with compact master manipulator," in $A C M$ SIGGRAPH, vol. 24, no. 4. ACM Press, 1990, pp. 165-170.

[24] A. Bloomfield, Y. Deng, J. Wampler, P. Rondot, D. Harth, M. McManus, and N. Badler, "A taxonomy and comparison of haptic actions for disassembly tasks," in VR'03. IEEE Computer Society, 2003, p. 225.

[25] K. V. Nesbitt, "A framework to support the designers of haptic, visual and auditory displays," in GOTHI'05, Saskatoon, Saskatchewan, Canada, 2005.
[26] M. K. D. Coomans and H. J. P. Timmermans, "Towards a taxonomy of virtual reality user interfaces," in $I V^{\prime} 97,1997$.

[27] J. Bertin, Semiology of Graphics, H. Wainer, Ed. The University of Wisconsin Press, 1983.

[28] J. Lohse, H. Rueter, K. Biolsi, and N. Walker, "Classifying visual knowledge representations: a foundation for visualization research," in VIS '90, A. E. Kaufman, Ed. Los Alamitos, CA, USA: IEEE Computer Society Press, 1990, pp. 131-138.

[29] J. P. Fritz and K. E. Barner, "Design of a haptic graphing system," in RESNA, 1996

[30] W. Yu, R. Ramloll, and S. Brewster, "Haptic graphs for blind computer users," in Workshop on Haptic Human-Computer Interaction, ser LNCS, S. Brewster and R. Murray-Smith, Eds., vol. 2058. SpringerVerlag Berlin Heidelberg, 2000, pp. 102-107.

[31] W. Yu, R. Ramloll, S. A. Brewster, and B. Riedel, "Exploring computer-generated line graphs through virtual touch," in ISSPA'01. IEEE Computer Society, 2001.

[32] T. Miller and R. Zeleznik, "The design of $3 \mathrm{~d}$ haptic widgets," in SI3D '99. ACM Press, 1999, pp. 97-102.

[33] R. Ramloll, W. Yu, S. Brewster, B. Riedel, M. Burton, and G. Dimigen, "Constructing sonified haptic line graphs for the blind student: First steps," in ACM SIGACCESS. ACM Press, 2000, pp. 17-25.

[34] W. Yu, K. Cheung, and S. Brewster, "Automatic online haptic graph construction," in EH'02, 2002, pp. 128-133.

[35] J. C. Roberts, K. Franklin, and J. Cullinane, "Virtual haptic exploratory visualization of line graphs and charts," in Electronic Imaging Conference, vol. 4660B, 2002, pp. 10-19.

[36] K. Franklin, "Non-visual data visualization: towards a better design," Ph.D. dissertation, Computer Science, University of Kent, 2007.

[37] S. Panëels and J. C. Roberts, "Haptic guided visualization of line graphs: pilot study," in HAID'07, poster and demo proceedings, L. Brown and T.-J. Jang, Eds., 2007, pp. 5-6.

[38] S. Panëels, J. C. Roberts, and P. J. Rodgers, "Haptic interaction techniques for exploring chart data," in HAID'09, ser. LNCS. SpringerVerlag Berlin Heidelberg, 2009, to appear.

[39] T. Way and K. Barner, "Automatic visual to tactile translation, part I: Human factors, access methods and image manipulation," in Trans. Rehab. Eng., vol. 5, no. 1. IEEE Computer Society, 1997, pp. 81-94.

[40] _ _ "Automatic visual to tactile translation, part II: Evaluation of the tactile image creation system," in Trans. Rehab. Eng., vol. 5, no. 1. IEEE Computer Society, 1997, pp. 95-105.

[41] W. Yu, K. Guffie, and S. Brewster, "Image to haptic data conversion: A first step to improving blind peoples accessibility to printed graphs," in $E H^{\prime} 01,2001$

[42] K. W. Brodlie, "Visualization techniques," in Scientific Visualization: Techniques and Applications, K. Brodlie, L. Carpenter, R. Earnshaw, J. Gallop, R. Hubbold, A. Mumford, C. Osland, and P. Quarendon, Eds. Springer-Verlag Berlin Heidelberg, 1992, ch. 3, p. 37.

[43] W. Yu and S. Brewster, "Comparing two haptic interfaces for multimodal graph rendering," in HAPTICS'02. Florida, USA: IEEE Computer Society, 2002, pp. 3-9.

[44] _ " "Multimodal virtual reality versus printed medium in visualization for blind people," in ACM SIGACCESS. Edinburgh, Scotland: ACM Press, 2002, pp. 57-64

[45] S. A. Wall and S. A. Brewster, "Tac-tiles: multimodal pie charts for visually impaired users," in NordiCHI '06. ACM Press, 2006, pp. $9-18$.

[46] W. Yu and S. A. Brewster, "Evaluation of multimodal graphs for blind people," Univers. Access Inform. Soc., vol. 2, no. 2, pp. 105-124, June 2003.

[47] S. A. Wall and S. A. Brewster, "Assessing haptic properties for data representation," in ACM CHI, vol. 2. ACM Press, 2003, pp. 858-859.

[48] S. Wall and S. Brewster, "Providing external memory aids in haptic visualisations for blind computer users," in ICDVRAT, 2004, pp. 157164.

[49] S. Wall, "Using more salient haptic cues in data visualisation," in $A C M$ CHI Workshop Hands-on Haptics. Portland, Oregon: ACM Press, 2005.

[50] S. Wall and S. Brewster, "Feeling what you hear: tactile feedback for navigation of audio graphs," in CHI 2006. ACM Press, 2006.

[51] D. K. McGookin, J. Kildal, and S. A. Brewster, "New views on haptic graph visualisation," in ACM CHI Workshop Hands-on Haptics. Portland, Oregon: ACM Press, 2005.

[52] G. L. Lohse, K. Biolsi, N. Walker, and H. H. Rueter, "A classification of visual representations," Communications of ACM, vol. 37, no. 12, pp. 36-49, 1994 
[53] W. Jeong and M. Gluck, "Multimodal bivariate thematic maps with auditory and haptic display," in ICAD'02. Kyoto, Japan: Advanced Telecommunications Research Institute (ATR), 2002.

[54] _ - "Multimodal geographic information systems: adding haptic and auditory display," JASIST, vol. 54, no. 3, pp. 229-242, Feb 2003.

[55] W. Jeong, "Multimodal trivariate thematic maps with auditory and haptic display," in ASIST, A. Grove, Ed., vol. 42, North Carolina, USA 2005.

[56] A. Gardiner and C. Perkins, "Feel the bunkers: Tactile maps for blind golfers," in ICA Commission on Tactile Maps and Graphics for Blind and Visually Impaired People: Needs, solutions, developments, Ljubljana, Slovenia, 1996, pp. 26-36.

[57] S. Jehoel, D. McCallum, J. Rowell, and S. Ungar, "An empirical approach on the design of tactile maps and diagrams: The cognitive tactualization approach," Br. J. Vis. Impair., vol. 24, no. 2, pp. 67-75, 2006

[58] J. Schneider and T. Strothotte, "Constructive exploration of spatial information by blind users," in ACM SIGACCESS. Arlington, Virginia, USA: ACM Press, 2000, pp. 188-192.

[59] P. Parente and G. Bishop, "Bats: The blind audio tactile mapping system," in ACMSE'03. Savannah, GA.: ACM Press, 2003.

[60] O. Lahav and D. Mioduser, "Multi-sensory virtual environment for supporting blind persons' acquisition of spatial cognitive mapping, orientation and mobility skills," in ICDVRAT'00, Alghero, Italy, 2000.

[61] _ _ "A blind person's cognitive mapping of new spaces using a haptic virtual environment," J. Res. Spec. Educ. Needs, vol. 3, no. 3, pp. 172177, 2003.

[62] — "Exploration of unknown spaces by people who are blind using a multi-sensory virtual environment," J. Spec. Educ. Tech., 2004.

[63] H. König, J. Schneider, and T. Strothotte, "Haptic exploration of virtual buildings using non-realistic haptic rendering," in ICCHP'OO. Vienna, Austria: Austrian Computer Society (OCG), 2000, pp. 377-384.

[64] C. Springsguth and G. Weber, "Design issues of relief maps for haptic displays," in $\mathrm{HCI}^{\prime} 03$, C. Stephanidis, Ed., vol. 4. Crete: Lawrence Erlbaum, 2003, pp. 1477-1481.

[65] C. Magnusson and K. Rassmus-Gröhn, "A dynamic haptic-audio traffic environment," in EH'04, 2004.

[66] L. Pokluda and J. Sochor, "Spatial haptic orientation for visually impaired people," in EG'03, I. Oakley, S. O'Modhrain, and F. Newell, Eds., vol. 2, 2003, pp. 29-34.

[67] _ _, "Spatial orientation in buildings using models with haptic feedback," in WHC'05. Pisa, Italy: IEEE Computer Society, 2005, pp. $523-524$.

[68] G. Jansson and P. Pedersen, "Obtaining geographical information from a virtual map with a haptic mouse," in ICC'05. The International Cartographic Association (ICA-ACI), 2005.

[69] H. König, J. Schneider, and T. Strothotte, "Orientation and navigation in virtual haptic-only environments," in Workshop on Guiding Users through Interactive Experiences: Usability Centred Design and Evaluation of Virtual 3D Environments, V. Paelke and S. Volbracht, Eds. Paderborn, Germany: C.LAB Publication, 2000, pp. 123-134.

[70] D. Chandler, Semiotics: the basics. Routledge, 2002.

[71] S. Gumtau, "Freshly squeezed touch into bits: towards the development of a haptic design palette," IJVR, vol. 9, no. 4, pp. 250-259, April 2006.

[72] K. MacLean and M. Enriquez, "Perceptual design of haptic icons," in EH'03, I. Oakley, O'Modhrain, and F. Newell, Eds., Dublin, Ireland, 2003.

[73] M. J. Enriquez and K. E. MacLean, "The hapticon editor: A tool in support of haptic communication research," in HAPTICS'03. Los Angeles, CA, USA: IEEE Computer Society, 2003, p. 356.

[74] C. Swindells, E. Maksakov, K. E. MacLean, and V. Chung, "The role of prototyping tools for haptic behavior design," in HAPTICS'06. Washington DC, USA: IEEE Computer Society, 2006.

[75] J. Y. C. Ng, J. C. F. Man, S. Fels, G. Dumont, and J. M. Ansermino, "An evaluation of a vibro-tactile display prototype for physiological monitoring," Anesthesia \& Analgesia, vol. 101, no. 6, pp. 1719-1724, Dec 2005.

[76] A. Chan, K. MacLean, and J. McGrenere, "Learning and identifying haptic icons under workload," Sensory perception and interaction research group, Tech. Rep. TR-2004-15, 2004.

[77] J. Pasquero, J. Luk, S. Little, and K. MacLean, "Perceptual analysis of haptic icons: an investigation into the validity of cluster sorted mds," in HAPTICS'06. Washington D.C., USA: IEEE Computer Society, 2006, p. 67.

[78] M. Enriquez, K. MacLean, and C. Chita, "Haptic phonemes: basic building blocks of haptic communication," in ICMI '06. Banff, Alberta, Canada: ACM Press, 2006, pp. 302-309.
[79] M. P. van Esch-Bussemakers and A. H. M. Cremers, "User walkthrough of multimodal access to multidimensional databases," in ICMI'04. ACM Press, 2004, pp. 220-226.

[80] J. C. Lee, P. H. Dietz, D. Leigh, W. S. Yerazunis, and S. E. Hudson, "Haptic pen: a tactile feedback stylus for touch screens," in UIST '04 ACM Press, 2004, pp. 291-294.

[81] B.-C. Lee, H. Park, J. Lee, and J. Ryu, "Tactile visualization with mobilear on a handheld device," in HAID'07, I. Oakley and S. Brewster, Eds., vol. LNCS, no. 4813. Springer-Verlag Berlin Heidelberg, 2007, pp. 11-21.

[82] C. Sjöström, "Using haptics in computer interfaces for blind people," in ACM CHI. ACM Press, 2001, pp. 245-246.

[83] — "Virtual haptic search tools - the white cane in a haptic computer interface," in AAATE'01. Ljubljana, Slovenia: IOS Press, Amsterdam, 2001.

[84] G. S. Lee and B. Hannaford, "Anisotropies of touch in haptic icon exploration," in IROS '03, vol. 3. IEEE Computer Society, 2003, pp. $2713-2717$

[85] S. A. Brewster and L. M. Brown, "Tactons: Structured tactile messages for non-visual information display," in AUIC'04. Dunedin, New Zealand: Australian Computer Society, 2004, pp. 15-23.

[86] E. Hoggan and S. Brewster, "New parameters for tacton design," in ACM CHI. ACM Press, 2007, pp. 2417-2422.

[87] H. A. H. C. van Veen and J. B. F. van Erp, "Tactile information presentation in the cockpit," in Workshop on Haptic Human-Computer Interaction, ser. LNCS, S. Brewster and R. Murray-Smith, Eds., vol. 2058. Springer-Verlag Berlin Heidelberg, 2000, pp. 174-181.

[88] L. M. Brown, S. A. Brewster, and H. C. Purchase, "A first investigation into the effectiveness of tactons," in WHC'05. Pisa, Italy: IEEE Computer Society, 2005, pp. 167-176.

[89] S. Töyssy, J. Raisamo, and R. Raisamo, "Telling time by vibration," in $E H^{\prime} 08$, ser. LNCS, M. Ferre, Ed., vol. 5024. Springer-Verlag Berlin Heidelberg, 2008, pp. 924-929.

[90] S. Ertan, C. Lee, A. Willets, H. Tan, and A. Pentland, "A wearable haptic navigation guidance system," in ISWC '98. IEEE Computer Society, 1998, pp. 164-165.

[91] S. Bosman, B. Groenendaal, J. W. Findlater, T. Visser, M. de Graaf, and P. Markopoulos, "Gentleguide: An exploration of haptic output for indoors pedestrian guidance," in Mobile HCI, ser. LNCS, vol. 2795 Springer-Verlag Berlin Heidelberg, 2003, pp. 358-362.

[92] C. Jansen, A. Wennemers, W. Vos, and E. Groen, "Flytact: A tactile display improves a helicopter pilot's landing performance in degraded visual environments," in EH'08, ser. LNCS, M. Ferre, Ed., vol. 5024. Springer-Verlag Berlin Heidelberg, 2008, pp. 867-875.

[93] A. H. Rupert, "An instrumentation solution for reducing spatial disorientation mishaps," IEEE Eng. Med. Biol. Mag., vol. 19, no. 2, pp. 71-80, March-April 2000.

[94] J. B. F. van Erp and H. A. H. C. van Veen, "Vibro-tactile information presentation in automobiles," in EH'01, 2001, pp. 99-104.

[95] J. B. F. van Erp, H. A. H. C. van Veen, C. Jansen, and T. Dobbins, "Waypoint navigation with a vibrotactile waist belt," ACM Trans. Applied Perception, vol. 2, no. 2, pp. 106-117, 2005.

[96] H. Z. Tan, R. Gray, J. J. Young, and R. Traylor, "A haptic back display for attentional and directional cueing," Haptics-e, vol. 3, no. 1, June 2003.

[97] R. W. Lindeman, Y. Yanagida, H. Noma, and K. Hosaka, "Wearable vibrotactile systems for virtual contact and information display," Virtual Real., vol. 9, no. 2, pp. 203-213, Dec 2006.

[98] L. A. Jones, B. Lockyer, and E. Piateski, "Tactile display and vibrotactile pattern recognition on the torso," Advanced Robotics, vol. 20 , no. 12 , pp. 1359-1374, 2006.

[99] N. Osawa, "Tactile glyphs for palpation of relationships," in $I V ' 06$ Washington, DC, USA: IEEE Computer Society, 2006, pp. 575-584.

[100] E. Hoggan, S. Anwar, and S. A. Brewster, "Mobile multi-actuator tactile displays," in HAID'07, I. Oakley and S. Brewster, Eds., vol. LNCS, no. 4813. Springer-Verlag Berlin Heidelberg, 2007, pp. 22 33.

[101] E. Hoggan, S. A. Brewster, and J. Johnston, "Investigating the effectiveness of tactile feedback for mobile touchscreens," in $\mathrm{CHI}$ '08. ACM Press, 2008, pp. 1573-1582.

[102] S. Mann, 'A historical account of 'wearcomp' and 'wearcam' inventions developed for applications in 'personal imaging,," in ISWC ' 97. IEEE Computer Society, 1997, pp. 66-73.

[103] V. G. Chouvardas, A. N. Miliou, and M. K. Hatalis, "Tactile displays: a short overview and recent developments," in ICTA'05, 2005.

[104] _ - "Tactile display applications: A state of the arts survey," in BCI'05, 2005 
[105] H. Z. Tan and A. Pentland, Tactual Displays for Sensory Substitution and Wearable Computers. Mahwah, NJ: LEA, 2001, ch. 18, pp. 579598.

[106] S. Brewster and R. Murray-Smith, Eds., Haptic Human-Computer Interaction First International Workshop (2000), ser. LNCS. SpringerVerlag Berlin Heidelberg, Aug 2001, vol. 2058

[107] K. Dobson, D. Boyd, W. Ju, J. Donath, and H. Ishii, "Creating visceral personal and social interactions in mediated spaces," in $A C M C H I$. ACM Press, 2001, pp. 151-152.

[108] B. S. Hoyle, J. M. Fowler, D. A. Waters, and D. J. Withington, "Development of the electronic guide cane for enhanced primary mobility for the vision impaired," in CVHI'04, 2004.

[109] S. Brewster and A. King, "An investigation into the use of tactons to present progress information," in Interact'05, M. F. Costabile and F. Patern, Eds. Rome, Italy: Springer-Verlag Berlin Heidelberg, 2005, pp. 6-17.

[110] J. C. Roberts and K. Franklin, "Haptic glyphs (hlyphs) - structured haptic objects for haptic visualization," in WHC'05, A. Bicchi and M. Bergamasco, Eds. IEEE Computer Society, 2005, pp. 369-374.

[111] D. Yi and V. Hayward, "Augmenting computer graphics with haptics for the visualization of vessel networks," in $P G^{\prime} 02$. IEEE Computer Society, 2002, p. 375.

[112] C. Jay, R. Stevens, R. Hubbold, and M. Glencross, "Using haptic cues to aid non-visual structure recognition," ACM Trans. Appl. Percept., vol. 5, no. 2, pp. 1-14, 2008.

[113] B. P. Challis and A. D. N. Edwards, "Weasel: a computer based system for providing non-visual access to music notation," ACM SIGCAPH, no. 66, pp. 1-12, 2000.

[114] L. L. Chu, "Using haptics for digital audio navigation," in ICME'O2, vol. 2. Lausanne, Switzerland: IEEE Computer Society, 2002, pp. 441-444.

[115] T. Beamish, K. MacLean, and S. Fels, "Designing the haptic turntable for musical control,' in HAPTICS'03. IEEE Computer Society, 2003.

[116] T. Beamish, K. Maclean, and S. Fels, "Manipulating music: multimodal interaction for djs," in ACM CHI. Vienna, Austria: ACM Press, 2004, pp. 327-334.

[117] R. M. Taylor II, W. Robinett, V. L. Chi, J. Frederick P. Brooks, W. V. Wright, R. S. Williams, and E. J. Snyder, "The nanomanipulator: a virtual-reality interface for scanning tunneling microscope," in $A C M$ SIGGRAPH. ACM Press, 1993, pp. 127-134.

[118] R. M. Taylor II, J. Chen, S. Okimoto, N. Llopis-Artime, V. L. Chi, J. Frederick P. Brooks, M. Falvo, S. Paulson, P. Thiansathaporn, D. Glick, S. Washburn, and R. Superfine, "Pearls found on the way to the ideal interface for scanned-probe microscopes," in Visualization '97. IEEE Computer Society, 1997, pp. 467-ff.

[119] R. M. Taylor II, "Practical scientific visualization examples," SIGGRAPH Comput. Graph., vol. 34, no. 1, pp. 74-79, 2000.

[120] F. P. Brooks Jr, M. Ouh-Young, J. J. Batter, and P. J. Kilpatrick, "Project grope - haptic displays for scientific visualization," in $A C M$ SIGGRAPH, vol. 24, no. 4. ACM Press, 1990, pp. 177-185.

[121] G. Sankaranarayanan, S. Weghorst, M. Sanner, A. Gillet, and A. Olson, "Role of haptics in teaching structural molecular biology" in HAP TICS'03. Los Angeles, CA, USA: IEEE Computer Society, 2003, pp. 363-367.

[122] L. J. K. Durbeck, N. J. Macias, D. M. Weinstein, C. R. Johnson, and J. M. Hollerbach, "Scirun haptic display for scientific visualization," in $P U G^{\prime} 98$, Dedham, MA, 1998.

[123] E. F. Wies, J. A. Gardner, M. S. O’Modhrain, C. J. Hasser, and V. L. Bulatov, "Web-based touch display for accessible science education," in Workshop on Haptic Human-Computer Interaction, ser. LNCS, vol. 2058. Springer-Verlag Berlin Heidelberg, 2000, pp. 52-60.

[124] R. S. Avila and L. M. Sobierajski, "A haptic interaction method for volume visualization,' in VIS '96, R. Yagel and G. M. Nielson, Eds. IEEE Computer Society, 1996, pp. 197-204.

[125] L. Y. Pao and D. A. Lawrence, "Synergistic visual/haptic computer interfaces," in Japan-USA-Vietnam RESCCE Workshop, 1998, pp. 155162.

[126] D. A. Lawrence, L. Y. Pao, C. D. Lee, and R. Y. Novoselov, "Synergistic visual/haptic rendering modes for scientific visualization," IEEE Comput. Graph. Appl., vol. 24, no. 6, pp. 22-30, 2004.

[127] D. A. Lawrence, C. D. Lee, L. Y. Pao, and R. Y. Novoselov, "Shock and vortex visualization using a combined visual/haptic interface," in VIS ’00. IEEE Computer Society, 2000, pp. 131-137.

[128] F. Infed, S. Brown, C. Lee, L. Lawrence, A. Dougherty, and L. Pao, "Combined visual /haptic rendering modes for scientific visualization," in ASME Int. Mech. Eng. Congress, Dynamic Systems and Control Div., vol. DSC-Vol. 67, Nashville, 1999, pp. 93-99.
[129] T. van Reimersdahl, F. Bley, T. Kuhlen, and C. H. Bischof, "Haptic rendering techniques for the interactive exploration of cfd datasets in virtual environments," in EGVE '03. Zurich, Switzerland: ACM Press, 2003, pp. 241-246.

[130] E. Mendez, S. Yoshida, H. Noma, R. W. Lindeman, Y. Yanagida, S. Masaki, and K. Hosaka, "Haptic-assisted guidance system for navigating volumetric data sets," in $W H C^{\prime} 05$. IEEE Computer Society, 2005, pp. 531-534.

[131] K. Lundin, M. Cooper, A. Persson, D. Evestedt, and A. Ynnerman, "Enabling design and interactive selection of haptic modes," Virtual Real., vol. 11, no. 1, pp. 1-13, 2007.

[132] K. L. Palmerius and C. Forsell, "The impact of feedback design in haptic volume visualization," in WHC'09. IEEE Computer Society, 2009, pp. 154-159.

[133] K. Kahol, P. Tripathi, T. McDaniel, and S. Panchanathan, "Rendering block diagrams accessible through audio-haptic interface," in CVAVI'05. IEEE Computer Society, 2005

[134] M. Horstmann, P. Blenkhorn, D. Crombie, S. Dijkstra, D. Evans, C. Hagen, O. Herzog, G. Ioannidis, A. King, and C. Schlieder, "Tedub: Automatic interpretation and presentation of technical diagrams for blind people," in CVHI'04, 2004.

[135] M. Lorenz and M. Horstmann, "Semantic access to graphical web ressources for blind user," in ISWC '04. Hiroshima, Japan: The Semantic Web Science Association (SWSA): online publication, 2004

[136] Z. B. Fredj and D. A. Duce, "Grassml: accessible smart schematic diagrams for all," in W4A'06. ACM Press, 2006, pp. 57-60.

[137] S. E. Krufka and K. E. Barner, "Automatic production of tactile graphics from scalable vector graphics," in ACM SIGACCESS. ACM Press, 2005, pp. 166-172.

[138] M. Kurze, "Rendering drawings for interactive haptic perception," in ACM CHI. ACM Press, 1997, pp. 423-430.

[139] - "Tguide: A guidance system for tactile image exploration," $J$. Behav. Inform. Tech, vol. 18, no. 1, pp. 11-17, 1999.

[140] R. G. Laycock, S. D. Laycock, and A. M. Day, "Haptic navigation and exploration of high quality pre-rendered environments," in VAST'06, Cyprus, 2006, pp. 17-24.

[141] H. Siirtola and E. Mäkinen, "Constructing and reconstructing the reorderable matrix," in IV'05, vol. 4. IEEE Computer Society, 2005 pp. 32-48.

[142] J. Kildal and S. A. Brewster, "Explore the matrix: Browsing numerical data tables using sound," in ICAD'05, 2005.

[143] _ , "EMA-Tactons: Vibrotactile external memory aids in an auditory display," in Interact'07, ser. LNCS, vol. 4663. Springer-Verlag Berlin Heidelberg, 2007, pp. 71-84.

[144] J. B. van Erp, I. Andrew, and J. Carter, "Iso's work on tactile and haptic interaction guidelines," in EH'06, 2006, pp. 467-470.

[145] J. C. Roberts, "Visualization equivalence for multisensory perception," IEEE Comput. Sci. Eng., vol. 6, no. 3, pp. 61-65, May 2004.

[146] J. C. Roberts and S. Panëels, "Where are we with haptic visualization?" in $W H C$ '07. IEEE Computer Society, 2007, pp. 316-323.

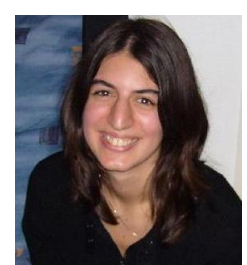

Sabrina Panëels is a $\mathrm{PhD}$ student at the School of Computing, University of Kent, UK. She received her MSc by Research (2005) from the University of Hull, UK and her engineering diploma (2005) from I.I.E, France, both in Computer Science. Her research interests include haptics, visualization and virtual reality.

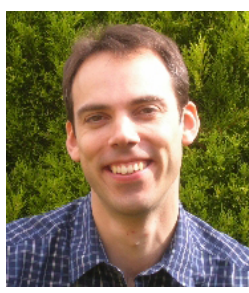

Jonathan C. Roberts is a senior lecturer at the School of Computer Science, Bangor University, U.K. He received his BSc (1992) and Ph.D. (1996) from the University of Kent, both in Computer Science. His research interests include visualization and VR; especially exploratory visualization, multiple views, haptic interfaces, visualization in Virtual environments, visualization reference models and Web-based visualization. $\mathrm{He}$ is the chair of Eurographics UK Chapter, Member of IEEE, ACM, and Eurographics societies. 